\title{
Global dynamics and diffusion in the rational standard map
}

\author{
Pablo M. Cincotta ${ }^{\mathrm{a}, \mathrm{b}, *}$, Carles Simóc \\ ${ }^{a}$ Grupo de Caos en Sistemas Hamiltonianos, Facultad de Ciencias Astronómicas y Geofísicas, Universidad Nacional de La Plata, La Plata, Argentina \\ ${ }^{\mathrm{b}}$ Instituto de Astrofisica de La Plata (CONICET), La Plata, Argentina \\ ${ }^{\mathrm{c}}$ Departament de Matemàtiques i Informàtica, Universitat de Barcelona, Barcelona, Spain
}

\section{A R T I C L E I N F O}

\section{Article history:}

Received 23 December 2019

Received in revised form 29 June 2020

Accepted 26 July 2020

Available online 8 August 2020

Communicated by G. Froyland

\section{Keywords:}

Area preserving maps

Rational standard map

Global dynamics

Chaotic diffusion

Shannon entropy

\begin{abstract}
A B S T R A C T
In this paper we study the dynamics of the Rational Standard Map, which is a generalization of the Standard Map. It depends on two parameters, the usual $K$ and a new one, $0 \leq \mu<1$, that breaks the entire character of the perturbing function. By means of analytical and numerical methods it is shown that this system presents significant differences with respect to the classical Standard Map. In particular, for relatively large values of $K$ the integer and semi-integer resonances are stable for some range of $\mu$ values. Moreover, for $K$ not small and near suitable values of $\mu$, its dynamics could be assumed to be well represented by a nearly integrable system. On the other hand, periodic solutions or accelerator modes also show differences between this map and the standard one. For instance, in case of $K \approx 2 \pi$ accelerator modes exist for $\mu$ less than some critical value but also within very narrow intervals when $0.9<\mu<1$. Big differences for the domains of existence of rotationally invariant curves (much larger, for $\mu$ moderate, or much smaller, for $\mu$ close to 1 than for the standard map) appear. While anomalies in the diffusion are observed, for large values of the parameters, the system becomes close to an ergodic one.
\end{abstract}

(C) 2020 Elsevier B.V. All rights reserved.

\section{Introduction}

One of the most studied area-preserving maps is the well known standard map (SM hereafter) introduced by Chirikov in $[1,2]$ as a representative model of a multiplet of interacting nonlinear resonances. In the last fifty years many studies deal with this system in order to understand several properties of twist mappings (see for instance [3-8] and references therein). It is defined as

$$
\bar{y}=y+K f(x), \quad \bar{x}=x+\bar{y}, \quad f(x)=\sin x,
$$

where $K$ is the so-called perturbation parameter and $x \in \mathbb{S}, y \in \mathbb{R}$ or, alternatively, both state variables are considered in $\mathbb{S}$. The SM can be seen as a discrete representation of a Hamiltonian flow with potential function $V(x)=\cos x$ such that $-d V / d x=f(x)$. But in order to have a Hamiltonian such that its time- $2 \pi$ map is close to the SM it is necessary to produce an instantaneous jump in $y$ followed by a suitable increase of the value of $x$. This can be achieved using a potential which is the product of $V(x)$ by a $2 \pi$ periodic $\delta$ distribution, to be denoted as $\delta_{2 \pi}$. Then the modified

\footnotetext{
* Corresponding author.

E-mail addresses: pmc@fcaglp.unlp.edu.ar (P.M. Cincotta), carles@maia.ub.es (C. Simó).
}

Hamiltonian has the form (see Appendix for details)

$$
H(y, x, t)=\frac{y^{2}}{4 \pi}+\frac{K}{2 \pi} \sum_{n=-\infty}^{\infty} \cos (x-n t) .
$$

The dynamics of the SM has been studied in a lot of works for many values of $K$. It is a nearly regular system for $K \ll 1$, small chaotic domains are created when increasing $K$, the transition to large chaos (rotationally invariant curves do not exist) occurs for $K \sim 1$ as well as the global chaotic dynamics when $K \gg 1$. In the nearly full chaotic regime small stability domains persist and inhibit the system to behave as ergodic, even for large values of $K$. The well known accelerator modes and other high-periodic solutions prevent the free diffusion leading to an anomalous transport process. In a broad sense it could be said that the dynamics of the SM is well known.

In this paper we investigate the full dynamics of a generalization of the SM, the so-called Rational Standard Map (RSM hereafter) using, instead of $f(x)$, a function $f(x ; \mu)$ which is no longer entire in $\mathbb{C}$ and which depends on a parameter $0 \leq \mu<1$, given by

$f(x ; \mu)=\frac{\sin (x)}{1-\mu \cos (x)}$,

This generalization of the SM was already considered in [9-11] where the map depends on an additional parameter which destroys the odd character of $f(x ; \mu)$. It could be regarded as an 
alternative representation of the non-linear map introduced, for instance, in [12] to investigate, in the case of a multidimensional model, the Arnol'd diffusion [13].

The main difference between the SM and the RSM, as we shall discuss in Section 2, is the appearance of the full harmonic spectrum in the corresponding Hamiltonian leading to a quite interesting dynamics. Letting $\mu \rightarrow 0$ the RSM reduces to the SM and we recover all the well known results.

A naive interpretation of this modified SM would be that the introduction of the denominator $1-\mu \cos x$ could enhance the perturbation parameter leading to a system where the chaotic regime should prevail even for $K<1$. As we shall see along this work, this expected behavior does not apply and the dynamics of the RSM turns out to be much more stable than the SM, even for $K \sim 1$ and moderate values of $\mu$. Moreover, we will show that the RSM presents noticeable differences with respect to the SM for a wide range of values of the perturbation parameter whenever $\mu \neq 0$. On the other hand even for moderate or small values of $K$ but $\mu$ close to 1 , the RSM is nearly ergodic.

Therefore throughout this paper we investigate in detail the dynamics of the RSM by means of analytical and numerical methods. Concretely in Section 2 beyond expansions of $H$ for the RSM, we give details on fixed and periodic points and accelerator modes and comment on the differences with the SM.

In Section 3 we look at the role of resonances in the global structure of the dynamics and at the domain of existence of rotationally invariant curves (RICs from now on). We also comment on the role played by values of the parameters for which homoclinic tangencies appear concerning when these invariant curves appear relatively close to the $x$ axis, and how they organize the amount of chaos for nearby parameters. We also look at the anomalies in the behavior of the diffusion due to accelerators modes and at the Shannon entropy. In all cases suitable numerical simulations allow to illustrate the different observed phenomena.

\section{The rational standard map}

The RSM generalizes the SM and for $y \in \mathbb{R}, x \in[0,2 \pi)$, it is defined as

$\bar{y}=y+K f(x ; \mu), \quad \bar{x}=x+\bar{y}, \quad f(x ; \mu)=\frac{\sin x}{1-\mu \cos x}$,

where $0 \leq \mu<1, K>0$. See for instance [9-11]. Models of multiharmonic SMs have been studied in several papers, like the seminal works of Ketoja and MacKay [14,15]. See also [16,17] and references therein.

The main difference between the RSM and the SM is the $\mathcal{O}(K)$ resonances set with coefficients which depend on $\mu$. Indeed, $f(x ; \mu)$ has as potential function,

$V_{0}(x ; \mu)=-\frac{1}{\mu} \ln (1-\mu \cos x)$,

$f(x ; \mu)=-\frac{d V_{0}(x, \mu)}{d x}, \quad \mu \neq 0$.

As it is shown in the Appendix, $V_{0}(x ; \mu)$ can be Fourier expanded as

$V_{0}(x ; \mu)=\sum_{l=1}^{\infty} C_{l}(\mu) \cos (l x)$,

where the constant term has been neglected and the expression for $C_{l}(\mu)$ is given in the Proposition included in Appendix. Recalling that the equations of motion leading to the map (2) involve the $2 \pi$-periodic $\delta_{2 \pi}(t)$ (see Appendix, Eq. (29))

$\dot{y}=K f(x ; \mu) \delta_{2 \pi}(t), \quad \dot{x}=\frac{y}{2 \pi}$,

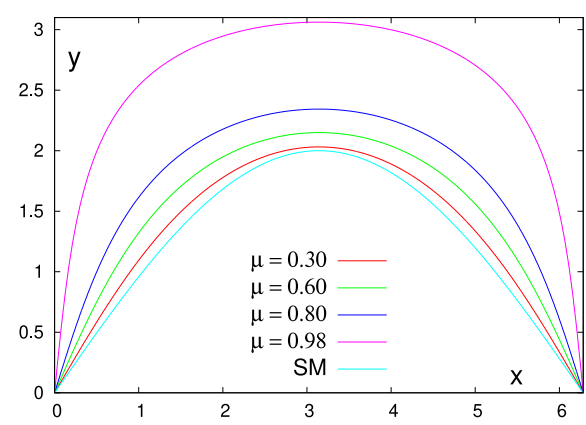

Fig. 1. Separatrix of the Hamiltonian $H_{K, \mu}(x, y)$ for several values of $\mu$, including $\mu=0$, for $K=1$.

the full potential satisfying (4) is $V(x, t ; \mu)=V_{0}(x ; \mu) \delta_{2 \pi}(t)$. Performing the product and by means of elementary trigonometric relations we arrive to the following Hamiltonian

$$
\begin{aligned}
& H(y, x, t)=\frac{y^{2}}{4 \pi}+\frac{K}{2 \pi} V(x, t ; \mu), \\
& V(x, t ; \mu)=\sum_{m=-\infty}^{\infty} \sum_{l=1}^{\infty} C_{l} \cos (l x+m t) .
\end{aligned}
$$

A simple comparison of the Hamiltonian leading to the RSM with the one of SM (1) reveals the differences between both functions. See also the Appendix for smooth modifications of $V(x, t ; \mu)$ such that can be integrated with standard integrators of ODE and the time- $2 \pi$ map is a good approximation of the RSM.

Although the potential (3) seems to be physically unrealistic, (5) shows that the Fourier expansion includes all the harmonics in $x$. The RSM represents a pendulum model with small oscillation frequency $\sqrt{K C_{1}(\mu)} / 2 \pi$ acted upon by a perturbation with a full Fourier spectrum which in fact is a more realistic scenario than the SM where only a single harmonic appears.

The separatrix of the Hamiltonian $H_{K, \mu}(x, y)=y^{2} / 2+K V_{0}(x ; \mu)$ (energy level for the maximum of $K V_{0}(x ; \mu)$ ) is shown in Fig. 1 for $K=1$ and different values of $\mu$ (to be compared with the manifolds of the origin for the RSM map to be shown in Fig. 8). The width of the separatrix, $(\Delta y)_{s}$, largely increases with $\mu$. For $x_{s} \neq 2 n \pi, K>0, \lim _{\mu \rightarrow 1}(\Delta y)_{s}=\infty$.

Next we present some simple facts about fixed or periodic points and accelerator modes. Using the differential map of the RSM

$$
\begin{aligned}
& \mathrm{D} \mathrm{RSM}=\left(\begin{array}{rr}
1+K f^{\prime}(x ; \mu) & 1 \\
K f^{\prime}(x ; \mu) & 1
\end{array}\right), \quad \text { being } \\
& f^{\prime}(x ; \mu)=\frac{\cos x-\mu}{(1-\mu \cos x)^{2}}
\end{aligned}
$$

it turns out that $\left(x_{0}, y_{0}\right)=(0,2 \pi m)$ is always unstable while for $\left(x_{0}, y_{0}\right)=(\pi, 2 \pi m)$ we get

$K<4(1+\mu)$

as stability condition, revealing that for $\mu \neq 0$ the stability border for the integer resonances is wider than in case of the SM.

We can look at stable points such that $y$ jumps, when we consider $y \in \mathbb{R}$, but they appear as fixed or periodic when we consider $y \bmod 2 \pi$ (accelerator modes) that satisfy

$K f\left(x_{0} ; \mu\right)=2 \pi n, \quad n \in \mathbb{Z} \backslash\{0\}, \quad-4<K f^{\prime}\left(x_{0} ; \mu\right)<0$.

Analytical approximations can be obtained in case of small $\mu$ and large $K$. Accelerator modes play an essential role in the observed anomalous diffusion in the SM (see for instance $[5,8]$ and references therein). 
In the limit when $\mu \rightarrow 0$ the $x_{0}$ values that satisfy (8) are close to the ones of the SM for $K \approx 2|n| \pi,|n| \geq 1$, $x_{0} \approx \pi / 2+\delta x_{0}, 3 \pi / 2-\delta x_{0}$ with $0<\delta x_{0} \ll 1$. Therefore $1-\mu \cos x_{0} \approx 1+\mu \delta x_{0} \approx 1$ and then the inequality in (8) reduces to $-4 / K+\mu \lesssim \cos x_{0}<\mu$, with $1 \ll K<4 / \mu$. So $\left|\cos x_{0}\right|$ should be small and from the above condition we assume that $\mathcal{O}(\mu) \sim \mathcal{O}\left(\left|\cos x_{0}\right|\right)$. Therefore we can expand $f\left(x_{0} ; \mu\right)$ and $f^{\prime}\left(x_{0} ; \mu\right)$ in powers of $\mu \cos x_{0}$ up to $\mathcal{O}\left(\mu^{2}\right)$ or $\mathcal{O}\left(\mu \cos x_{0}\right)$ and thus the stability condition (8) becomes

$4 \pi^{2} n^{2}<K^{2}\left(1+\mu^{2}\right)<4 \pi^{2} n^{2}+16$.

In the sequel we use, eventually, some scaled variables. Concretely we keep using $x$ for the value modulus $2 \pi, y$ denotes either the value in $\mathbb{R}$ or modulus $2 \pi$ (being clear depending on the context). We introduce

$k=\frac{K}{2 \pi}, \quad u=\frac{x}{2 \pi}, \quad v=\frac{y}{2 \pi}$.

\section{Fixed points and accelerator modes.}

In terms of the parameter $k$ the stability condition reads

$n^{2}<k^{2}\left(1+\mu^{2}\right)<n^{2}+\frac{4}{\pi^{2}}$,

which is similar to the domain of existence of accelerator modes in the SM, just replacing $k^{2}$ by $k^{2}\left(1+\mu^{2}\right)$. Therefore we observe that for a given value of $\mu \neq 0$, but small, the RSM presents accelerator modes for slightly smaller values of $k$ than in case of the SM $(\mu=0)$. However a more general expression of the range of existence of the $n$th accelerator mode for a given value of $\mu \in[0,1)$ can be derived (see below).

It is interesting to observe from (7), with $K=2 \pi k$, that for a given $k$ the integer resonances are stable when $\mu>\mu_{c}(k)=$ $\pi k / 2-1$ provided that $k<4 / \pi(\mu<1)$. Therefore, for instance in case of $k$ like in (10), $k \approx|n|$, the latter condition holds for $k \approx 1$ and thus if $\mu>\pi / 2-1 \approx 0.571$, the phase space of the RSM for these $(\mu, k)$ values would present large domains of stable motion, i.e., the integer resonances.

On the other hand for $k=|n|$ and from (10), valid for small $\mu$, accelerator modes of period 1 seem to exist whenever $\mu<$ $2 /(|n| \pi)$. Therefore as long as $k$ increases these periodic solutions are restricted to very small values of $\mu$, for instance $0 \leq \mu \lesssim$ 0.2122 for $k=3$. Anyway, very narrow stability intervals appear for relatively small $k$ and large values of $\mu$. This is our next goal.

Using variables as in (9) we rewrite the RSM in (2) as

$$
\bar{v}=v+k \hat{f}(u ; \mu), \quad \bar{u}=u+\bar{v}, \quad \hat{f}(u ; \mu)=\frac{\sin (2 \pi u)}{1-\mu \cos (2 \pi u)}
$$

Let us consider a given accelerator mode such that the point $\left(u^{*}, 0\right)$ jumps to $\left(u^{*}, n\right)$ for a given $\mu$ and a suitable value of $k$. The minimal value of $k$ for which this happens appears when $k=k^{(1)}=n\left(1-\mu^{2}\right)^{1 / 2}$, i.e. $n$ divided by the maximum of $\hat{f}(u ; \mu)$. From the derivative of $f$ given in (6), this maximum is attained at $u^{*}$ such that $\cos \left(2 \pi u^{*}\right)=\mu$.

We consider $u^{*} \lesssim 1 / 4$ and for the symmetric point $1-u^{*}$ the results that we mention are affected by this symmetry. Looking at that point and $v=0(\bmod 1)$, it is a fixed point with a double eigenvalue equal to 1 . Increasing $k$ a bifurcation occurs and two fixed points appear when taking $v(\bmod 1)$. One on the left, hyperbolic, and one on the right, elliptic. Around the elliptic point there are invariant curves which are broken at some distance from the fixed point giving rise to Cantori. The iterates of initial points lying in a chaotic zone can enter inside the Cantori, remain there for long time, due to the steepness of the invariant curves inside them, until they escape from the Cantori. After each iterate

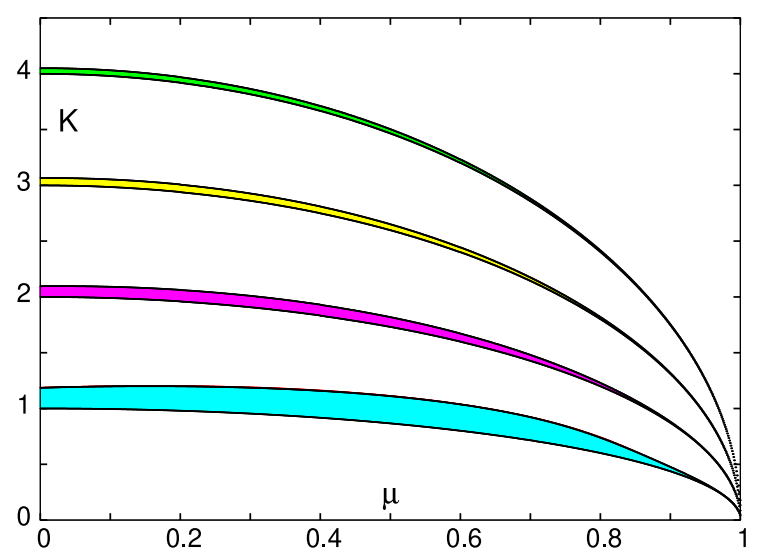

Fig. 2. Stability intervals in the parameter space $(\mu, k)$ for the accelerator modes obtained numerically for $n=1, \ldots, 4$ (from bottom to top). (For interpretation of the references to color in this figure legend, the reader is referred to the web version of this article.)

inside the Cantori $v$ increases by $\approx n$, producing an anomaly in the diffusion. See [5], for instance.

Increasing again $k$ we get a value such that the elliptic point has changed to parabolic and has a double eigenvalue equal to -1 . Then a new bifurcation is produced, giving rise to an hyperbolic point with reflection, an eigenvalue less than -1 , and a period-two elliptic orbit. Therefore invariant curves arise including inside the period-two elliptic orbit and the hyperbolic point and also Cantori around them, which play the same role as before. Hence, accelerator modes keep existing after this second bifurcation. It occurs when $k \hat{f}(u ; \mu)=n$ and $k \hat{f}^{\prime}(u ; \mu)=-4$. Both equations can be written as polynomials in $k, \mu$ and $\cos (2 \pi u)$. The resultant after eliminating the terms in $\cos (2 \pi u)$ is

$$
\begin{aligned}
& \frac{\pi^{2}}{4} k^{4}-\mu \pi k^{3}+\left(\frac{2 \mu^{2}-1}{4} n^{2} \pi^{2}+\mu^{2}-1\right) k^{2} \\
& +\left(\mu-\mu^{3}\right) n^{2} \pi k+\left(\mu^{4}-\mu^{2}\right) n^{4} \frac{\pi^{2}}{4}=0 .
\end{aligned}
$$

The value of $k$ associated to the bifurcation, for given values of $\mu$ and $n$, is the unique real positive root of (12). Denote this root as $k^{(2)}$ that will depend on $\mu$ and $n$ as it does $k^{(1)}$. Thus accelerator modes exist for given values of $\mu$ and $n>0$ which are centered at $\left(u^{*}, 0\right)$ with $u^{*}<1 / 2$ and, due to the antisymmetry of the RSM they appear in $u=1-u^{*}$ with $n<0$.

The amplitude $\Delta k:=k^{(2)}-k^{(1)}$ also depends on $(\mu, n)$. Replacing in (12) $k$ by $k^{(1)}+\Delta k$, using $k^{(1)}=n\left(1-\mu^{2}\right)^{1 / 2}$ we get a equation of degree 4 for $\Delta k$ with coefficients that depend on $\mu$ and $n$. Though easy, the corresponding expression is longer than (12). For $n$ large, the dominant terms are the ones of degree 0 and 1 , and thus an approximation for $\Delta k$ can be derived,

$\Delta k \approx \frac{2}{\pi^{2}} \frac{\left(1-\mu^{2}\right)^{3 / 2}}{n}$.

In particular the maximal relative error of this approximation for $\mu \in[0,1)$ is below 0.1 or 0.01 for $n>7$ and $n>64$, respectively. Note that if we use (10) to estimate $\Delta k$ for small $\mu$, it leads to $\Delta k \approx 2\left(1-\mu^{2}\right) /\left(\pi^{2} n\right)$, close to (13) if $\mu \ll 1$.

As mentioned, for $k>k^{(2)}(\mu, n)$ accelerator modes still exist. The range of $k$ can be obtained numerically. A simple method is to take a point very close to the unstable manifold of the related hyperbolic point with reflection and check if the iterates are confined in a vicinity of the fixed point when using $v(\bmod 1)$ and a large number of iterates. Fig. 2 shows for $\mu \in[0,1)$ and $n=$ $1,2,3,4$ (from bottom to top) the ranges $\left[k^{(1)}(\mu, n), k^{(2)}(\mu, n)\right]$ 
in different colors and where the black lines correspond to the extreme values. For small $\mu$ (say, up to $\mu \approx 0.25$ ) the amplitudes are inversely proportional to $n$. In the case $n=1$ we have also added in red the range of stability observed numerically for $k>$ $k^{(2)}(\mu, n)$. It is very narrow and hard to see. This has been also computed for the other values of $n$ but the ranges are narrower.

It is worth to note that the values of $k \hat{f}^{\prime}(u ; \mu)=-4$ at $k^{(2)}$, are very close to -4.085 when all the invariant curves around the accelerator mode are destroyed for $n=2,3$ and 4, except in a narrow range near $\mu=1$. In the case $n=1$ the same is true except in the range, roughly $\mu \in(0.3,0.8)$, where the values increase up to -4.0475 to decrease again near -4.085 .

\section{Period 2 points.}

Now let us consider the periodic points of period 2, that is when $x_{0} \leftrightarrow x_{1}$. First we look at the RSM in $\mathbb{T}^{2}$. From (2) it is easy to show that for $y \in[0,2 \pi)$, the phase values satisfy

$f\left(x_{0} ; \mu\right)+f\left(x_{1} ; \mu\right)=0$,

whose solutions are $x_{1}=-x_{0}, y_{1}=-y_{0}($ both $\bmod 2 \pi)$ or the trivial one $x_{0,1}=0, \pi, y_{0}=y_{1}=\pi$. In the first case $\left(x_{1}=-x_{0}, y_{1}=-y_{0}\right)$ the coordinates of the periodic point $\left(x_{0}, y_{0}\right)$ should satisfy $(\bmod 2 \pi)$

$4 x_{0}+K f\left(x_{0} ; \mu\right)=0, \quad y_{0}=2 x_{0}$.

The stability condition for these period two orbits, from the product of the corresponding differentials, is

$-4<K^{2} f^{\prime}\left(x_{0} ; \mu\right) f^{\prime}\left(x_{1} ; \mu\right)+2 K\left(f^{\prime}\left(x_{0} ; \mu\right)+f^{\prime}\left(x_{1} ; \mu\right)\right)<0$,

that in case of $f^{\prime}\left(x_{0} ; \mu\right)=f^{\prime}\left(x_{1} ; \mu\right)$ reduces to the second relation in (8) which applies when $x_{0}=-x_{1}$.

It is clear from (15) that one has to solve the equation,

$E(x, K, \mu)=4 x+K \frac{\sin x}{1-\mu \cos x}=0(\bmod 2 \pi)$,

for $x_{0}$ as a function of $K, \mu$, discarding the trivial solutions $x_{0}=0$, $x_{0}=\pi$ which correspond to fixed points.

For concreteness we bound our domain of interest to $K<4$. According to (7), for larger values of $K$ there exist values of $\mu$ such that the fixed point at $(\pi, 0)$ becomes unstable and when $K$ increases, keeping $\mu$ fixed, it gives rise to a 2-periodic orbit having one of the points in $x_{0}$, satisfying $E(x, K, \mu)=2 \pi$ and $y_{0}=2 x_{0}-2 \pi$.

For $\mu=0$ it is clear that the unique solution of $4 x+$ $K \sin (x) /(1-\mu \cos x)=2 \pi$, with $x \in(0, \pi / 2)$ for $0<K<$ 4 , decreases monotonically when $K$ increases. Increasing $\mu$ this behavior subsists until a large value of $\mu, \mu^{*}$, for which there exists a value $K^{*}$ of $K$ such that $4 x+K^{*} \sin (x) /\left(1-\mu^{*} \cos x\right)=2 \pi$ has a triple zero.

To look for these values we use the equations $E=2 \pi, E^{\prime}=$ $0, E^{\prime \prime}=0$. From $E^{\prime \prime}=0$, skipping non-zero factors, we get $\mu$ as a function of $x$, and from $E^{\prime}=0$ we can write $K$ as a function of $x$ and $\mu$ :

$\mu(x)=\frac{\cos x+\sqrt{\cos ^{2} x+8}}{4}, \quad K=-4 \frac{(1-\mu \cos x)^{2}}{\cos x-\mu}$,

and thus $E(x, K, \mu)$ reduces to an equation which only depends on $x$

$x+\frac{(1-\mu(x) \cos x) \sin x}{\mu(x)-\cos x}-\frac{\pi}{2}=0$.

It has the unique solution $x^{*} \approx 0.5606513881$ and therefore $\mu^{*} \approx 0.9498521661, K^{*} \approx 1.4860378253$.

For $\mu^{*}<\mu<1$ there are ranges of $K$ with three 2-periodic solutions, say $x_{0,1}<x_{0,2}<x_{0,3}$ and the corresponding values of $y: y_{0, i}=2 x_{0, i}, i=1,2,3$. Let us denote as $\left[K^{a}(\mu), K^{b}(\mu)\right]$

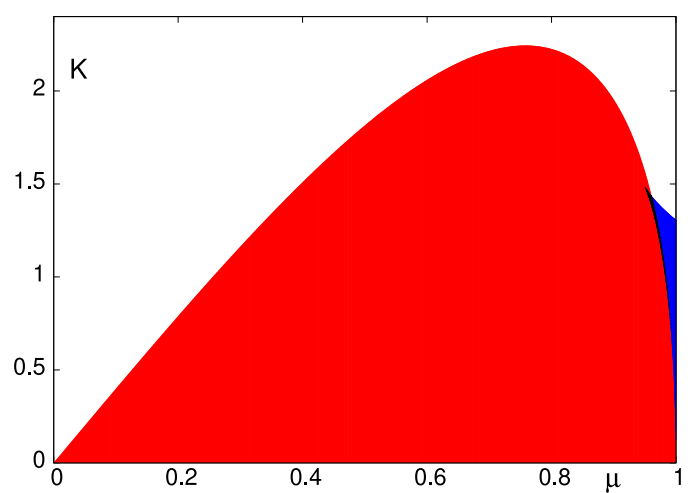

Fig. 3. Parameter space $(\mu, K)$ where (15) has only one stable solution is depicted in red; a triple zero at $\mu^{*} \approx 0.9498, K^{*} \approx 1.4860$ lying at the end of the black region inside the red one; while three roots $\left(x_{0, i}, i=1,2,3\right)$ exist, the ones labeled by $i=1,3$ are stable in the black zone while in the blue region only $x_{0,3}$ is stable. (For interpretation of the references to color in this figure legend, the reader is referred to the web version of this article.)

the range of existence of these three solutions for a given value of $\mu$. For $\mu=\mu^{*}$ we have $K^{a}=K^{b}=K^{*}$. When $\mu$ increases towards $1, K^{a} \rightarrow 0$ while $K^{b}$ approaches the value $K^{b} \approx 1.305$. At $K=K^{a}$ the solutions with $i=1,2$ are created by an elliptichyperbolic bifurcation. When $\mu \rightarrow 1$, defining $\delta=1-\mu \rightarrow 0$, the value of $K^{a}$ and the corresponding coordinate $x$ satisfy $x=$ $\sqrt{2 \delta}+\mathcal{O}(\delta), K=2 \pi x+\mathcal{O}\left(x^{2}\right)$. For every $\mu$ in the above mentioned range the solution $\left(x_{0,3}, y_{0,3}\right)$ is stable in the range $\left[K^{a}, K^{b}\right]$, the $\left(x_{0,2}, y_{0,2}\right)$ is unstable in the same range and $\left(x_{0,1}, y_{0,1}\right)$ is stable in the same range for $\mu$ close to $\mu^{*}$ and then it becomes stable only in a small range close to $K^{a}$, with a width range that tends to zero as $\mu \rightarrow 1$.

If we look at the full domain $\mu \in[0,1), K \in(0,4]$ for the set of values for which there is at least one stable solution of this type, we find a large domain which is connected. It is bounded by $K=0$, by a smooth curve whose tangent at $(0,0)$ has slope 4 , as it is easy to derive, and has a quadratic maximum at $\mu \approx$ $0.757696, K \approx 2.24437$. Then it decreases until it reaches the values of $K^{b}$ mentioned above. Fig. 3 illustrates all this stability analysis in parameter space for period two trajectories.

On the other hand, for the trivial solutions at $y_{0}=y_{1}=$ $\pi, x_{0}=0, x_{1}=\pi$, for $\mu \ll 1$ the centers of the semi-integer resonances appear. In this case $f^{\prime}\left(x_{0} ; \mu\right)=1 /(1-\mu), f^{\prime}\left(x_{1} ; \mu\right)=$ $-1 /(1+\mu)$ and from (16) being $K>0, \mu<1$, we get

$4 \mu<K<2(1+\mu)$,

showing that the semi-integer resonances of the RSM are stronger than in the SM, their centers are stable even for $K>2$ provided that $\mu>0$. Note that another difference in the behavior of the semi-integer resonances of the RSM with respect to the SM is that the $K$ values are bounded from below. Thus, even for small $\mu$, always exists a narrow interval $0<K<4 \mu$ where the two fixed points at $y_{1}=y_{2}=\pi, x_{0}=0, x_{1}=\pi$ are hyperbolic.

If instead we consider the RSM in the cylinder the condition (14) should be replaced by $f\left(x_{0} ; \mu\right)+f\left(x_{1} ; \mu\right)=2 \pi l, l \in \mathbb{Z}$ and thus much more possibilities of two periodic solution or accelerator modes are present. In a similar fashion it would be possible to find solutions of period 3, 4 and so on.

\section{Numerical results}

Several of the estimates given in Section 2 are for small $\mu$ and just for some particular periodic orbits. Therefore this section is devoted to investigate by numerical means the dynamics of the 

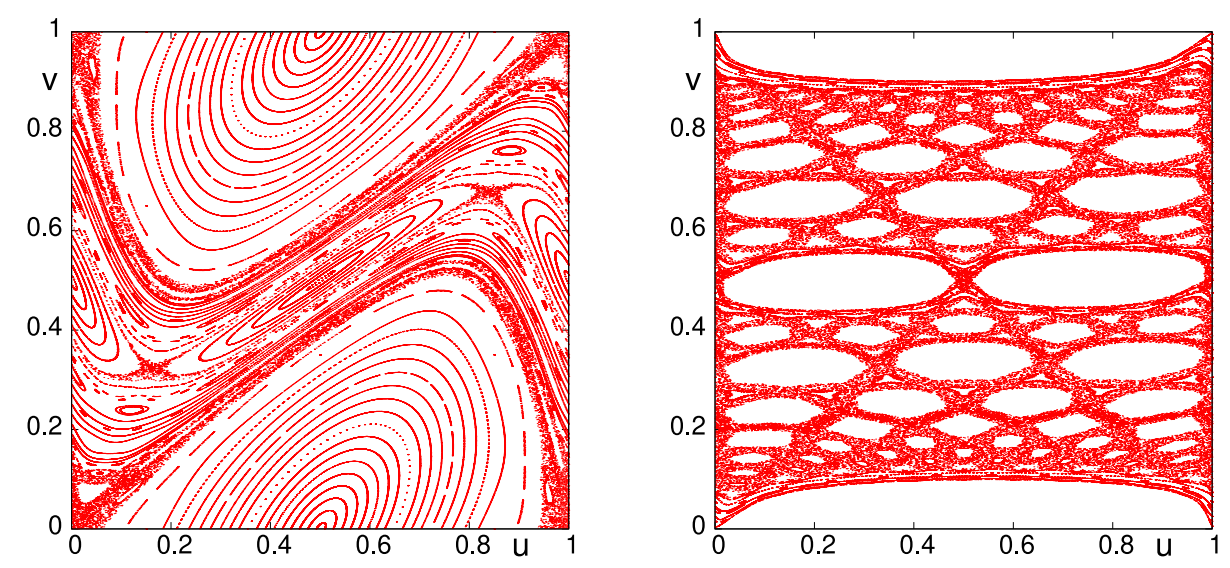

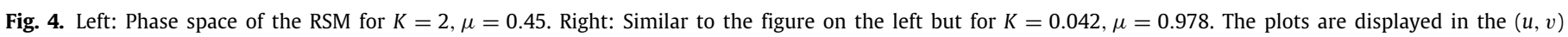
variables.

RSM. In what follow in this Section we use either $(u, v)$ or $(x, y)$ as variables, and either $K$ or $k$ as parameter. It is clear from the context.

Fig. 4 illustrates the phase space of the RSM for different values of $K$ and $\mu$, on the left $K=2, \mu=0.45$ while $K=0.042, \mu=$ 0.978 on the right panel. As discussed in the previous section, the parameter $\mu$ plays a crucial role in the dynamics of the RSM. Indeed, for relatively large values of $K$ (in comparison with the $\mathrm{SM}$ ) and moderate values of $\mu$ the motion is mostly stable, chaos is almost restricted to the chaotic layers around the separatrix of the main resonances. Moreover, as it can be seen in the left plot, an invariant curve that intersects $x=0$ and $x=1$ at $y \approx 0.2$ confines the motion close to the resonance $y=0$, the fixed point at $(x, y)=(1 / 2,1 / 2)$ is stable accordingly to (17) $(\mu<K / 4)$ and thus the integer and semi-integer resonances do not overlap. On the other hand, for a small value of $K$ but large $\mu$, as shown on the right plot, the $(1 / 2,1 / 2)$ fixed point becomes hyperbolic leading to two new elliptic fixed points near $(1 / 4,1 / 2),(3 / 4,1 / 2)$ and a comparatively large stability domain appears. For these particular values of the parameters, the system looks globally chaotic although the main high order resonances are not destroyed by overlap. At least solutions up to period 10 are clearly distinguishable as small (or not so small) stability islands as well as their associated homoclinic tangles (see below). It is a curious fact that all the chaotic dynamics seen in the right plot can be obtained taking an initial condition on $(0,0.07)$ and performing a very large number of iterates.

Using the Hamiltonian (5) the coefficient $C$ of a given harmonic is $(K /(2 \pi))$ times the corresponding value of $C_{l}$ (given in the Appendix). The half-amplitude of the resonance is $2 \sqrt{C}$. It is checked that for $K=2, \mu=0.45$ these half-amplitudes exceed 0.01 up to the fifth harmonic, while for $K=0.042, \mu=0.978$, the half-amplitudes exceeding 0.01 go up to the tenth harmonic. These results agree with the resonances that can be clearly seen in the phase space of RSM given in Fig. 4. After these harmonics the successive half-amplitudes decrease in an exponential way, of the form $\approx \exp (-0.76 l)$ in the first case and $\approx \exp (-0.14 l)$ in the second one.

\subsection{Transition to chaos}

In this subsection we focus on the transition from mostly stable motion to global chaos, when a major overlap of resonances takes place. While the stability border by means of the overlap criterion of different resonances could be derived from the Hamiltonian (5), several restrictions appear. It is an easy task to compute the amplitudes of each resonance, $C_{l}$, but, on the other hand, the overlap criterion does not include the distortion of the separatrix due to resonance interaction as well as the width of the chaotic layer around the latter. Therefore, in order to get an accurate stability border we perform numerical experiments.

Let us consider $0 \leq \mu<0.999$ and $0.001 \leq K<2.5$ both parameters with step 0.001 and an ensemble of 10 initial conditions within a fundamental domain of the unstable manifold at the fixed point $(0,0), W_{0}^{u}$, at distances from the origin less than 0.001 . We iterate them first up to $10^{7}$ and if for a given value of the pair $(K, \mu)$ and $N_{i t e r}<10^{7}$ one has $|v| \geq 1$ the computation is stopped since a transition to global chaos occurs, the system moves freely between the integer resonances.

On the other hand, for those values of the action such that after $10^{7}$ iterates still one has $|v|<1$, presumably a RIC $\mathcal{C}$ exists that intersects $u=0,1$ at some value $|v|=v^{*}$ with $0<v^{*}<1 / 2$ since $\hat{f}(u ; \mu)$ is antisymmetric. Therefore we look for the values of the action $v=v_{m}$ whenever $u<0.001$ or $1-u<0.001$ (for some particular values of $K, \mu$ we take $u<10^{-4}, 1-u<10^{-4}$ ). In case $\left|v_{m}\right|>1 / 2, \mathcal{C}$ does not exist, these nearby initial conditions though look bounded up to $10^{7}$, they will not be confined and for larger times they will escape. After this procedure, the initial ensemble gives evidence of confinement for about $1.23 \times 10^{6}$ of the $2.5 \times 10^{6}$ values of $(K, \mu)$ used in the simulations.

In order to refine the border of stability in parameter space, let $(i, j)=(1000 K, 1000 \mu)$ be the parameter indexes for those values of $(K, \mu)$ for which the system seems to be stable. For a given pair $(i, j)$, define the set $I_{i j}=[i-1, i+1] \times[j-1, j+1]$. If an initial ensemble with parameter indexes in $I_{i j}$ is not confined, then we take again the parameter indexes $(i, j)$ and the 10 initial conditions are iterated up to $10^{9}$. In case the action values remain bounded, i.e, $\left|v_{m}\right|<1 / 2$ we assume that for these values of the parameters the ensemble is confined. This procedure is repeated for each $I_{i j}$ and finally we obtain near $1.22 \times 10^{6}$ values of $(K, \mu)$ for which one has numerical evidence of the existence of a RIC $\mathcal{C}$.

Fig. 5 presents in red the region of the $(K, \mu)$ space, $\Sigma_{b}$, where the numerical evidence suggests that the iterates of initial data taken on $W_{0}^{u}$ remain bounded by an invariant curve. We also include in the figure the stability interval for the two-periodic orbits given by (17). Note that the border of stability of the RSM is not smooth (fractal structures are clearly observed, see for instance [14-17] for related discussions), a large stability domain appears for $K$ values at both sides of the line $K=4 \mu$ when $\mu<0.6$. At $\mu=0$ we obtain the expected value of $K=K_{c} \approx$ 0.9716354 (Greene's value) and only for $\mu \gtrsim 0.75$ the stability border in $K$ lies below $K_{c}$. From these results it becomes evident that the change in the stability properties of the period 2 orbit starting at $(1 / 2,1 / 2)$ plays a crucial role in shaping this border. 


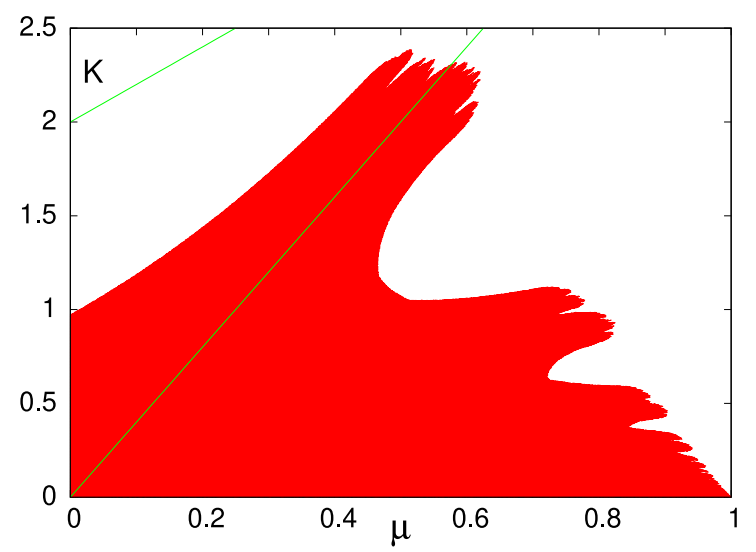

Fig. 5. Parameter space region, $\Sigma_{b}$, for which an initial ensemble of nearby initial conditions on $W_{0}^{u}$ is bounded by a RIC around the integer resonance $v=0$. The green lines corresponds to the stability interval $4 \mu<K<2(1+\mu)$ for the centers of the semi-integer resonances.

We can compare these results for the RSM with the ones that one obtains for the SM with two harmonics, say SM2h. For instance with the ones presented as an example in [17]. There the function $f$ in SM2h depends on a parameter $\alpha$ and is given by $f(x, \alpha)=\cos (2 \pi \alpha) \sin (x)+\sin (2 \pi \alpha) \sin (2 x)$. Due to symmetries it is enough to consider $\alpha \in(-1 / 4,1 / 4]$. For $\alpha=0$ one recovers the classical SM and for $\alpha=1 / 4$ one recovers also the classical SM with $K, x, y$ replaced by $2 K, 2 x, 2 y$. According to [17] the maximal values of $K$ for which a RIC exist are $K=$ $K_{c} \approx 0.9716354$ for $\alpha=0, K=K_{c} / 2$ for $\alpha=1 / 4$, and the extreme values appear for $(\alpha, K) \approx(0.0232,1.8887)$, the global maximum of $K$, and for $(\alpha, K) \approx(-0.1620,0.3298)$, the global minimum. In contrast, for the RSM the maximum appears for $(\mu, K) \approx(0.515,2.384)$, a value of $K$ much larger than the one for the SM2h. For that value of $\mu$ there are relevant harmonics in $f(x, \mu)$ (say, with amplitude $>10^{-3}$ ) until the sixth one. On the other hand for a given $\mu$ the maximal value of $K$ tends to 0 as $\mu \rightarrow 1$. As an example, the number of relevant harmonics for $\mu=0.999$ is 169 .

For all $(K, \mu)$ in $\Sigma_{b}$, a RIC $\mathcal{C}$ exists and intersects the $v$ axis at $v_{M} \leq 1 / 2$ (see Fig. 4 left panel). Thus we focus on those invariant curves that confine the motion close to the separatrix of the resonance $v=0$, that is when the chaotic layer is very thin and thus the amount of unstable chaotic motion is small. Considering the numerical simulation that leads to Fig. 5, we look for the maximum values of the action whenever $u<0.001$ and such that $v_{M} \ll 1 / 2$, that is the largest action value where $\mathcal{C}$ intersects the $v$ axis very close to the origin. Adopting the intervals $0.1 / 2^{l+1}<2 \pi v_{M} \leq 0.1 / 2^{l}, l=0,1, \ldots, 4$ and $0<$ $2 \pi v_{M} \leq 0.1 / 2^{5}$ the results are shown in Fig. 6 (left panel). Notice the particular filament structure that appears around what it seems to be invariant curves in parameter space that minimize the width of the chaotic layer at $u=0$. As far as the authors know this has not been observed in the SM2h.

Another comparison of the RSM with the SM2h (which includes the SM) is the following. To produce Fig. 4 right one can take initial points with $u=0$ and $M$ equispaced values of $v$ (e.g. $M=100$ ). Then one can do $N$ iterates for each of them (e.g. $N=10^{6}$ ) and plot every a large prime number (like 113). The orbits such that some iterate, if we consider $v \in \mathbb{R}$, goes away from $[0,1]$ are neglected. In this way Fig. 4 right can be produced. The used $(K, \mu)$ parameters are at a distance $\approx 0.01$ from the border of $\Sigma_{b}$.

The same method has been applied to more than 1000 values of the parameters $(K, \alpha)$ of the SM2h inside the domain of existence of RICs and close to the boundary. There are major differences with respect to the RSM like:

- The domains associated to the different resonances are near horizontal in Fig. 4 right, while in the SM2h have a shape like $a+b \sin (2 \pi u)$ or $a+b \sin (4 \pi u)$ for suitable values of $a$ and $b$. Furthermore in the SM2h case the domain associated to the period 2 islands is larger than in the RSM, while the size of the higher order periodic islands is smaller, much smaller for period 7 or higher and almost not visible for period 9 or higher. This is due to the fact that for the RSM there are many harmonics of relatively large size, while in the SM2h only two harmonics play a role, except if $\alpha$ is a multiple of $1 / 4$.

- In the RSM case the RICs are located very close to $v=0$ and $v=1$, and no more such curves exist. In the SM2h the lower and upper ones are not so close to $v=0$ or $v=1$ and many other appear for intermediate cases like, typically, the ones which appear before and after the resonant zone of period 2.

- For the SM2h one detects periodic points with one of the points in $u=0$ which have islands of visible size. This type of islands are almost invisible in the RSM case. The fact that at $u=0$ the differential of RSM contains terms of the form $1+K /(1-\mu)$ plays a relevant role.

In order to understand this curious structure we proceed as follows. For a given $\mu>\mu_{0}>0$ we look for the value of $v_{M}$ for a given $K$, say $K_{0}$, which is much smaller than the values of $v_{M}$ corresponding to the eight neighboring $K<K_{0}$ and $K>K_{0}$ values using the lattice in $(K, \mu)$ described before. In other words, we search in parameter space for those values of $(K, \mu)$ for which the closest $\mathcal{C}$ to the separatrix of the resonance $v=0$ appears. The results are given in Fig. 6 (right panel) where one can see points which give evidence of the existence of several smooth curves which follow the filament pattern observed in the left panel. It turns out that this particular structure in $\Sigma_{b}$ is indeed due to the presence of these "minimal" invariant curves. Later we shall look for geometrical reasons of this filament pattern.

Just to complement the above results and letting $0.01<K<$ 6.4 and $\mu$ in the same interval as before, we compute the largest Lyapunov exponent and we look for the region of the parameter space where the fraction of chaos, $\sigma$, is small. Given a grid of $400 \times 400$ values of the parameters in the above defined interval and after $2 \times 10^{4}$ iterates we get the results shown in Fig. 7 left panel, where red corresponds to $0.01<\sigma<0.1$, green to $0.001<\sigma<0.01$ and so on. We observe that a nearly stable, integrable motion in parameter space takes place along these filaments, revealing that the curves shown in Fig. 6 left panel are indeed invariant curves that bound the motion close to the separatrix of the integer resonance. The small amount of chaos is thus mostly confined to a small neighborhood around the separatrix of the resonance $y=0$.

\section{Stable and unstable manifolds.}

Let us focus now on the homoclinic tangle of the integer resonance. Since the motion near any $y=2 m \pi$ resonances for $\mu$ not too large can be reduced to a driven pendulum, as discussed in the previous section, then very close to the separatrix the change in the energy due to the perturbation is given by the Melnikov integral (see [2] for instance)

$A_{m}(\lambda)=\int_{-\infty}^{\infty} d t \cos \left(\frac{m}{2} \varphi(t)-\lambda t\right), \quad \varphi(t)=4 \arctan \mathrm{e}^{t}$,

for any real $m$ and where the integration domain involves the full motion on the unperturbed separatrix, i.e. from the unstable fixed points $(x, y)=(0,0)$ and $(2 \pi, 0)$ at $t=\mp \infty$ respectively. Since 

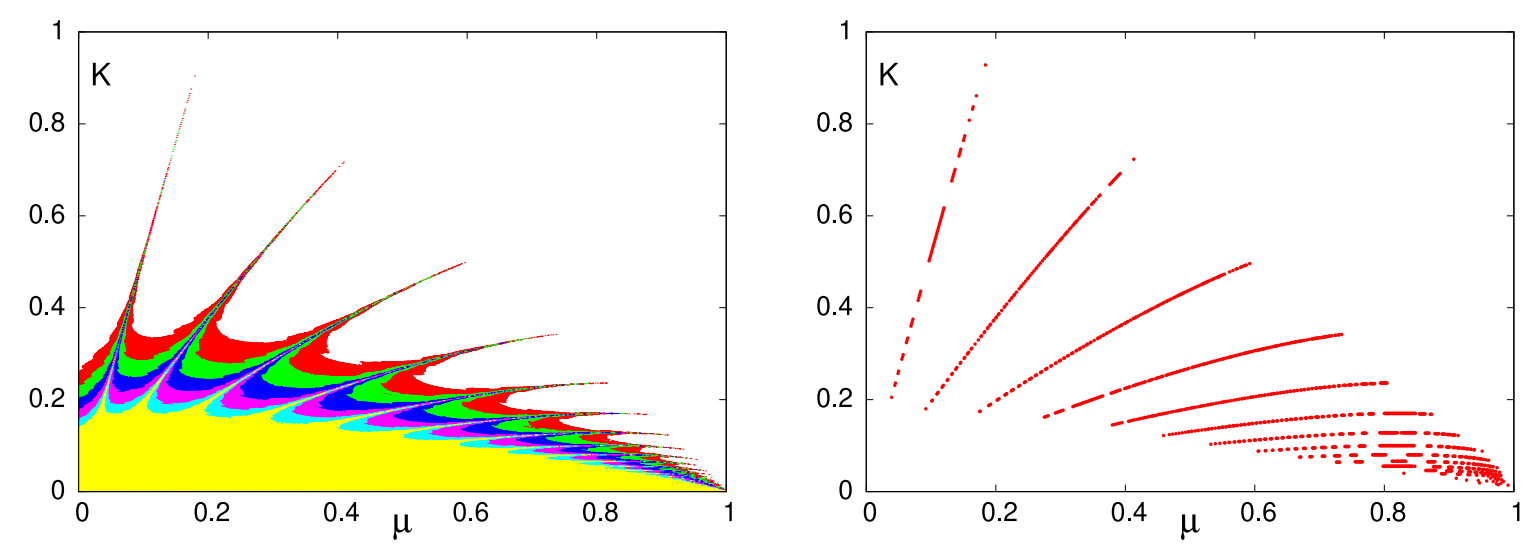

Fig. 6. Left panel: Values of $0.1 / 2^{l+1}<2 \pi v_{M} \leq 0.1 / 2^{l}, l=0,1, \ldots, 4$ and $0<2 \pi v_{M} \leq 0.1 / 2^{5}$ in the parameter space, where the palette color is given in red for $0.05<2 \pi v_{M} \leq 0.1$ and similarly in green, blue, magenta, cyan and yellow respectively. Right panel: Evidence of the existence of smooth curves in parameter space $(K, \mu)$ for which $\mu>\mu_{0}>0$ and $v_{M}$ for a given $K=K_{0}$ is much smaller than the values of $v_{M}$ corresponding to the eight neighboring, $K<K_{0}$ and $K>K_{0}$ values. (For interpretation of the references to color in this figure legend, the reader is referred to the web version of this article.)
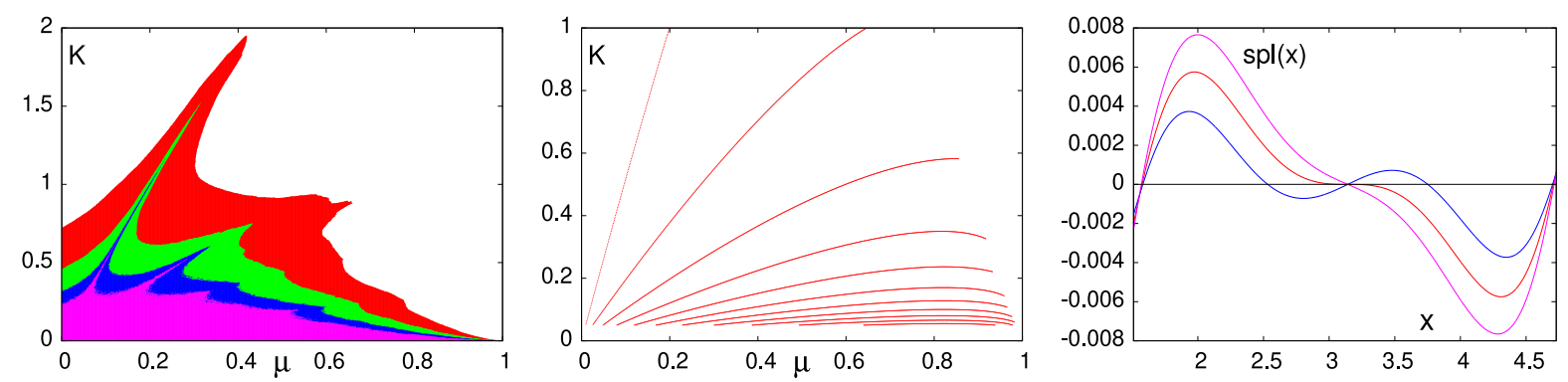

Fig. 7. Left: Fraction of chaos, $\sigma$, in parameter space $(\mu, K)$, in red $0.01<\sigma<0.1$, in green $0.001<\sigma<0.01$, in blue $0.0001<\sigma<0.001$, in magenta $\sigma<0.0001$. Center: Values of $\left(\mu_{0}, K_{0}\right)$ in parameter space where the invariant manifolds $W_{0}^{u}$ and $W_{0}^{u}$ are tangent at the central homoclinic point, located on $x=\pi$. Right: The splitting function against $x$ within a fundamental domain for $\mu_{0}=0.4, K=K_{0}=1.89062177$ and $K=1.88,1.90$ in red, blue and magenta respectively. (For interpretation of the references to color in this figure legend, the reader is referred to the web version of this article.)

the above integral is symmetric with respect to $t=0(x=\pi)$, the contribution of the unstable manifold $\bar{W}_{0}^{u}$ from $x=0$ to $x=\pi$ to the above integral is the same as that of the stable manifold $\bar{W}_{0}^{s}$ arriving at $x=2 \pi$ from $x=\pi$. Then at $x=\pi$ the perturbed whiskers $W_{0}^{u}$ and $W_{0}^{s}$ coincide in an homoclinic point $h$.

Les us consider some pair $(K, \mu)$ in $\Sigma_{b}$ shown in Fig. 5 , for which the motion is bounded by a RIC. We compute the linear approximation to $W_{0}^{u}$ and $W_{0}^{s}, w_{0}^{u}, w_{0}^{s}$ respectively, considering distances to the origin less than $10^{-18}$ (the computations are carried out in quadruple precision). Since $W_{0}^{u}$ and $W_{0}^{s}$ intersect at $x=\pi$, we numerically obtain this homoclinic point $h$ at $(x, y)=\left(\pi, y_{h}\right)$ and by using the tangent map (direct and inverse in case of $W_{0}^{u}$ and $W_{0}^{s}$, respectively) the eigenvectors at the origin $\left(w_{0}^{u}, w_{0}^{s}\right)$, are transported to the homoclinic point $h$. Let $\left(w^{u}, w^{s}\right)$ be the normalized eigenvectors at $h$, then $\left\|w^{u} \times w^{s}\right\| \approx \alpha$, where $\alpha$ is the angle between $w^{u}$ and $w^{s}$ at $x=\pi$ and it is clear that $w_{0}^{u}, w_{0}^{s}, w^{u}, w^{s}, \alpha$ depend on $K$ and $\mu$.

Defining the splitting function $\operatorname{spl}(x)=W^{u}(x)-W^{s}(x)$ (looking at the manifolds as graphs, at least locally), then $\alpha=\left.\operatorname{spl}^{\prime}(x)\right|_{x=\pi}$, where prime denotes derivative with respect to $x$. For a given $\mu=\mu_{0}$ we look for those values of $K=K_{0}$ for which $\alpha(\pi)=0$, i.e, where the splitting function presents an extreme value at $x=\pi$ and therefore at this particular homoclinic point $\operatorname{spl}(x)$ has at least a double zero. Therefore the amplitude of the splitting function is expected to be small.

The results for those values of the parameters in $\Sigma_{b},\left(K_{0}, \mu_{0}\right)$, where $\alpha=\alpha\left(K_{0}, \mu_{0}, \pi\right)=0$ are shown in Fig. 7 center panel. It is interesting to notice that the values of the parameters for which a tangency between $W^{u}$ and $W^{s}$ at the homoclinic point occurs are identical to those $(K, \mu)$ shown in Fig. 6 at the right for the "minimal" curves. Both results provide then stronger evidence that when $(K, \mu) \in \Sigma_{b}$ invariant curves very close to the homoclinic tangle of the integer resonance actually exist. See at the end of this subsection for a concrete example.

In the same direction, we also compute the splitting function and we observe that, indeed, its amplitude in a fundamental domain of the homoclinic tangle around $x=\pi$ is quite small, as Fig. 7 right panel illustrates for some special values of the parameters. In the figure $\operatorname{spl}(x)$ for $K=K_{0}=1.89062177$ (in red) corresponds to a point of the first curve from above in the figure at the center panel for $\mu=0.4$. For these particular values of $(K, \mu)$, as expected $\operatorname{spl}(\pi)=0$, where $x=\pi$ is a triple zero for $K=K_{0}$ while it becomes a single zero when $K=1.90$ with $\operatorname{spl}^{\prime}(\pi)<0$. In general, when varying $K$ away from the critical value $K_{0}$ such that $K \in\left(K_{0}-\Delta K, K_{0}+\Delta K\right)$ with $0<\Delta K \ll 1$, the triple zero at $x=\pi$ bifurcates to three simple zeros, one of them also at $x=\pi$ while the remainder appear at both sides of the latter, as the curve with $K=1.88$ shows. For different values of $\left(K_{0}, \mu_{0}\right)$ and closer (in $\left.K\right)$ the results are similar to the ones presented here, the most significant differences are the amplitudes of the splitting function and the size of $\Delta K$ when considering parameter values on different curves $\alpha=\alpha\left(K_{0}, \mu_{0}, \pi\right)=0$. Indeed, $\Delta K$ and the amplitude of the splitting function decrease in $\sim 10^{-2}$ and $\sim 10^{-3}$ respectively, when $\left(K_{0}, \mu_{0}\right)$ are taken in two neighboring curves.

In Fig. 8 we display for $K=1$ and different values of $\mu$ the beginning of the upper branches of the unstable/stable manifolds $\left(W^{u}, W^{s}\right)$ of the origin under the RSM. There are significant changes with respect to the separatrices of the related Hamiltonian system, displayed in Fig. 1. The values of $\mu$ considered are 

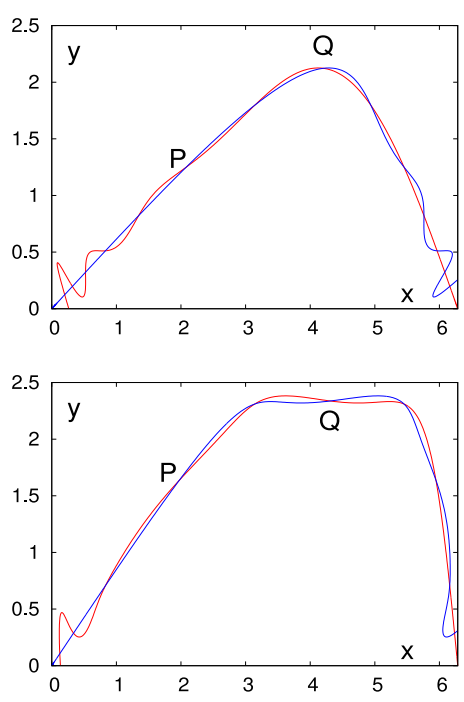
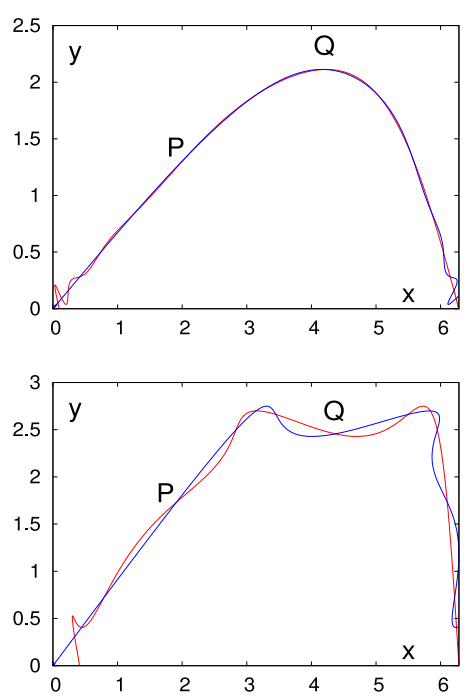
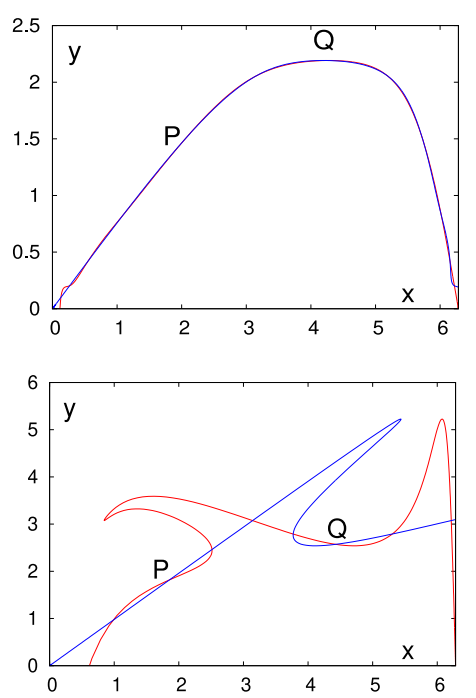

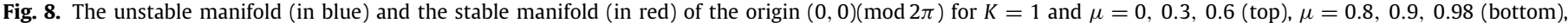
from left to right. (For interpretation of the references to color in this figure legend, the reader is referred to the web version of this article.)

the same as the ones in Fig. 1 with the addition of $\mu=0.9$ to see how significant changes between $\mu=0.8$ and $\mu=0.98$ start to appear.

If we compare with Fig. 5 we check that for $\mu=0$ one can clearly see the splitting. This is related to the fact that $K=1$ is larger than Greene's value for $\mu=0$. On the other hand $K=1$ for $\mu=0.3$ is well inside the red domain in Fig. 5 and for $\mu=0.6$ is still inside but close to the boundary. For these two values of $\mu$ the splitting is rather small. Between, say, $x=\pi / 2$ and $x=3 \pi / 2$ the manifolds seem visually coincident. For $\mu=0.8$ the value $K=1$ lies outside the intervals of existence of RICs and the splitting is quite visible again, of the order of magnitude of the case $\mu=0$. On the other hand for $\mu=0.9$ and $\mu=0.98$ we are far and very far, respectively, from the boundary of the red domain in Fig. 5.

The points marked as $P$ are homoclinic points and we denote as $Q$ the image of $P$ under the RSM. The arc from $P$ to $Q$ is a fundamental domain. In the case $\mu=0.8$ there are two homoclinic points in a fundamental domain (i.e., there is an additional homoclinic between $P$ and $Q$, as it should be at least, because the map is not only conservative but it is also orientation preserving). But for $\mu=0.9$ and, a fortiori, for $\mu=0.98$ there are four homoclinic points in such domain. For a value of $\mu$ between $\mu=0.8$ and $\mu=0.9$ a cubic tangency of $W^{u}$ and $W^{s}$ appears at one of the homoclinic points, like it is shown in Fig. 7 right. This produces the creation of two additional homoclinic points in a fundamental domain.

All these results explain the ones shown in Fig. 5. For instance the upper invariant curve in Fig. 7 center leads to the structure of $\Sigma_{b}$ at both sides of the line $K=4 \mu$. In conclusion, for values of the parameters in $\Sigma_{b}$, that is when no significant overlap of resonances takes place, the dynamics of the RSM could be assumed to be well represented by a nearly integrable system.

As an example of the role of the homoclinic tangency we have taken a value of $(\mu, K)$ in the third line, from top to bottom, of the central plot in Fig. 7 . For $\mu=0.5$ one has $K \approx 0.43906$. For these values of the parameters we computed a large number of iterates starting at a point, close to the origin, in $W^{u}$. It shows a small chaotic domain. Then we took initial points on the $x$ axis and in the $y$ axis, whose orbits give evidence of being invariant curves. On the other hand we use the same value of $\mu$, but changing $K$ tor 0.43 and 0.45 . In both cases the iterates of a point in $W^{u}$ show a comparatively larger chaotic domain as Fig. 9 reveals. Similar facts have been checked for other points in the curves of Fig. 7 , center.

\subsection{The dynamics of the RSM for large $K$}

In this subsection we investigate the dynamics of the RSM for $K \geq 2 \pi(k \geq 1)$ since according to (10) obtained for small $\mu$, accelerator modes corresponding to one periodic solutions (when we look at them in $\mathbb{T}^{2}$ ) are present at $k \approx|n|$.

In the SM for large values of the perturbation parameter (see for example $[4-7,18,19])$, periodic solutions and/or accelerator modes appear at particular values of the parameter $k$ such that when reducing the map to the torus $\mathbb{T}^{2}$ they show up as small stability islands embedded in the large chaotic sea. Except for integer or semi-integer values of $k$, the SM approximates to a random system, so the variance of the action grows linearly with time, the diffusion being normal and the diffusion coefficient is well approximated by the theoretical expected one in this stochastic limit as it was shown in for instance [5]. Accelerator modes at $k=n, n+1 / 2$ lead to a linear variation of the action with time, while the dynamics is confined near an accelerator mode, and thus superdiffusion occurs, the variance scaling with a square law with time. When we take into account that the iterates of a set of initial conditions spend some time near an accelerator mode, then escape from it and behave in a random way for some iterates, can be captured again by an accelerator mode, etc., the long term values of the variance increase as $t^{b}$ for some $b \in(1,2)$.

It is well known that if we use $v$ and $k$ as in (11), and we assume that the successive values of $v$ are uncorrelated, the diffusion coefficient, defined as the linear rate of evolution of the mean square value $\left\langle\Delta v^{2}\right\rangle$, takes the value $D_{S M}(k)=k^{2} / 2$ if $\mu=0$. In the general case one has the following result.

Proposition. For the RSM with parameters $k, \mu$ and assuming random motion, the theoretical diffusion coefficient is given by

$D_{R S M}(k, \mu)=k^{2} \frac{1}{1-\mu^{2}+\sqrt{1-\mu^{2}}}$.

Proof. As defined,

$D_{R S M}(k, \mu)=\left\langle\Delta v^{2}\right\rangle=k^{2} \frac{1}{2 \pi} \int_{0}^{2 \pi} \frac{\sin ^{2} x}{(1-\mu \cos x)^{2}} d x$

and letting aside the factor $k^{2}$, we need to evaluate the integral in (19). Using the integrals

$\int_{0}^{\pi} \frac{d x}{\left(1-2 a \cos x+a^{2}\right)^{2}}, \quad \int_{0}^{\pi} \frac{\cos 2 x d x}{\left(1-2 a \cos x+a^{2}\right)^{2}}$, 

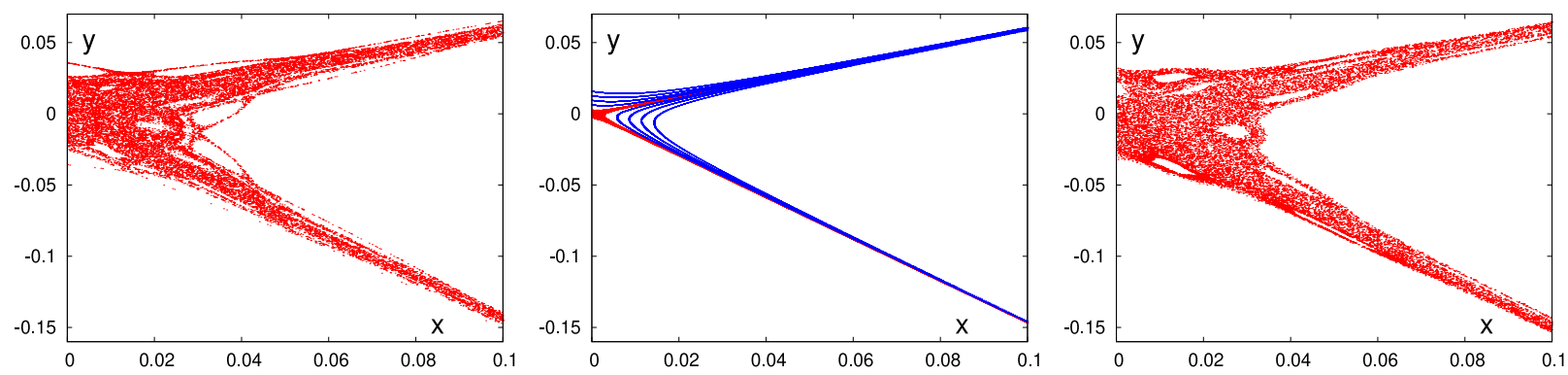

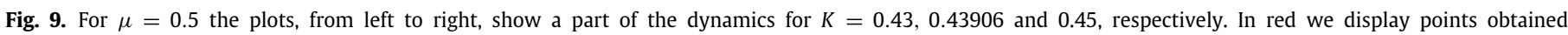

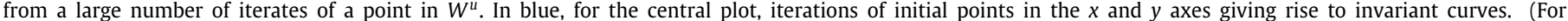
interpretation of the references to color in this figure legend, the reader is referred to the web version of this article.)

as given in [20] pp. 391-392, formula 3.616(2/10), and $a=\mu /(1+$ $\sqrt{1-\mu^{2}}$ ), one obtains (18).

Clearly $D_{R S M} \rightarrow \infty$ when $\mu \rightarrow 1$. To be more precise, setting again $\delta=1-\mu$ we get

$D_{R S M}(k, 1-\delta)=k^{2}\left(\frac{1}{\sqrt{2 \delta}}-1+\mathcal{O}(\sqrt{\delta})\right)$

when $\delta$ approaches zero.

For the diffusion numerical experiments we consider $10^{5}$ nearby initial conditions in a fundamental domain of $W^{u}$ at the origin with a maximum distance of $10^{-8}$. We iterate them up to a final time $t=N=10^{7}$ for each value of $k$ with $0<k \leq 5$ with a step $\Delta k=0.0005$ and three particular values of $\mu$. For every value of $k$ the ensemble variance $\left\langle\Delta v^{2}\right\rangle(t)$ is computed and it is sampled at equispaced values of $t$ with $\Delta t=1000$. Then for each $k$ we look for a power law of the form

$\left\langle\Delta v^{2}\right\rangle(k)=D(k) t^{b(k)}$.

The results are shown in Fig. 10, both for the exponent $b(k)$ and the coefficient $D(k)$, for three representative values of $\mu$. For every $k$ the fit is done looking for a least squares linear relation $\ln \left(\left\langle\Delta v^{2}\right\rangle(t)\right)=\ln D+b \ln t$. In the right plot the theoretical value for the diffusion coefficient for $\mu=0.95$ given by (20) is also included just for comparison.

We observe that for comparatively small $\mu$ departures from normal diffusion appear at $k \approx|n|,(2|n|+1) / 2$ while for large $\mu$, this is only observed when $k<1$, consistent with the discussion given after Eq. (10). Indeed, in case $k \approx 1$ one-period accelerator modes exist whenever $\mu<2 / \pi \approx 0.63$ and in very narrow intervals around large values of $\mu$ as Fig. 2 reveals. Beyond this value, the centers of the integer resonances are stable just for $k \approx 1$ and when reducing the map to the torus, these resonances should be the only relevant stability domains. For larger values of $k$ and $\pi k / 2-1<\mu<1$ the integer resonances are destroyed and thus no appreciable stable motion is expected in phase space of the RSM (see below for a more detailed discussion).

When considering a large ensemble of $n$ initial conditions rather than a single one, the value of $\left\langle\left(\Delta^{n} v\right)^{2}\right\rangle$ cannot be easily averaged since expressions of the form $k f(2 \pi(u+v+k f(2 \pi(u+$ ...))) appear leading to the observed oscillations in the computed values of the diffusion coefficient. This effect is also present in the SM (see [5]) and since in this case $f=\sin (x)=\sin (2 \pi u)$, the above expression can be expanded in $k$ by means of sums of Bessel functions and therefore the numerical values of the diffusion coefficient can be corrected. This explains the oscillation in the red and green curves when $k$ increases.

Deviations from the expected value of the exponent $b \leq 2$ at the values of $k=|n|$ are due to several factors being the most relevant: (i) roundoff errors when $v$ takes very large values, say $v>10^{6}$, while $f(2 \pi u) \sim \mathcal{O}(1)$; (ii) a finite number $N$ of iterates induces a lack of balance between those trajectories in the ensemble that are trapped by a given accelerator mode and those that escape. In the limit when $N \rightarrow \infty$ one expects that the balance holds. In other words, assuming that for a relatively small number of iterates most of the points are not captured by an accelerator mode, then a relevant fraction of them is captured and remains near the accelerator for a large number of iterates. Thus the fit to determine $b$ and $D$ can lead to values of $b$ larger than 2 but at the same time $D$ is rather small. The plot in Fig. 10 right gives also evidence of this second fact.

In order to illustrate this let us consider $\mu=0.5$ and $k=$ 1.835 where we obtained an exponent $b \approx 2.08$ (see Fig. 10 left). Repeating the procedure to determine $b$ but considering an ensemble of $10^{6}$ nearby initial conditions and $N=10^{9}$ iterates for all of them we get $b \approx 2.73$. How to explain these unexpected results?

The answer is clear: this can be due to roundoff errors. Assume that for given $(k, \mu)$ values some iterate of an initial condition approaches an accelerator mode passing through a hole of a Cantor set, moving there slowly, when we use (11) (mod 1), until it escapes from the vicinity of the mode also through a hole of a Cantor set. It can happen that in some (exact) iterate the point moves in phase by a rather small quantity (say, of the order of $10^{-10}$ ). But if $v$ is very large (say, of the order of $10^{8}$ or larger) when we compute $\bar{u}$ as $u+\bar{v}$ and reduce mod 1 to have it in $[0,1)$ many digits are lost. Say, of the order of one half if we work in double precision. That is, we only keep, say, the first 8 decimal digits. It can happen then that these digits for $(\bar{u}, \bar{v})$ coincide with the ones of $(u, v)$. Due to roundoff the point is seen as a fixed point and it never escapes from the vicinity of the accelerator mode.

To decrease the roundoff errors one can use the scaled variables in (9) and the formulation in (11) but we replace the variable $v \in \mathbb{R}$ by the sum of an integer $p$ and the decimal part of $v$ in $[0,1)$, that we denote again as $v$. Then we express the RSM as

$v^{*}=v+k \hat{f}(u ; \mu), \quad u^{*}=u+v^{*}, \quad \bar{u}=u^{*}-$ floor $\left(u^{*}\right)$,

$\bar{v}=v^{*}-\operatorname{floor}\left(v^{*}\right), \quad \bar{p}=p+\operatorname{floor}\left(v^{*}\right)$.

Note that it is essential to keep $p$ if we are interested in the variance of $v$ (the scaled $y$ ). Otherwise we can skip it.

Proceeding in this way and using (22) instead of (11) for the values of $(k, \mu)$ that gave this strange behavior, we get an exponent $b \approx 1.285$. This implies an exponent for the related increase of the standard deviation which is $1.285 / 2$, close to the values obtained in [5] for different examples with the SM.

Around these values of $\mu=0.5, k=1.835$ the accelerator mode corresponding to $|n|=2$ appears, and we investigated the dynamics of the RSM using (22) and the variables $u, v$. In fact, for $\mu=0.5$ this accelerator mode appears when $k=|n|\left(1-\mu^{2}\right)^{1 / 2}=$ $\sqrt{3}$. The bifurcation gives rise to an elliptic point that becomes 

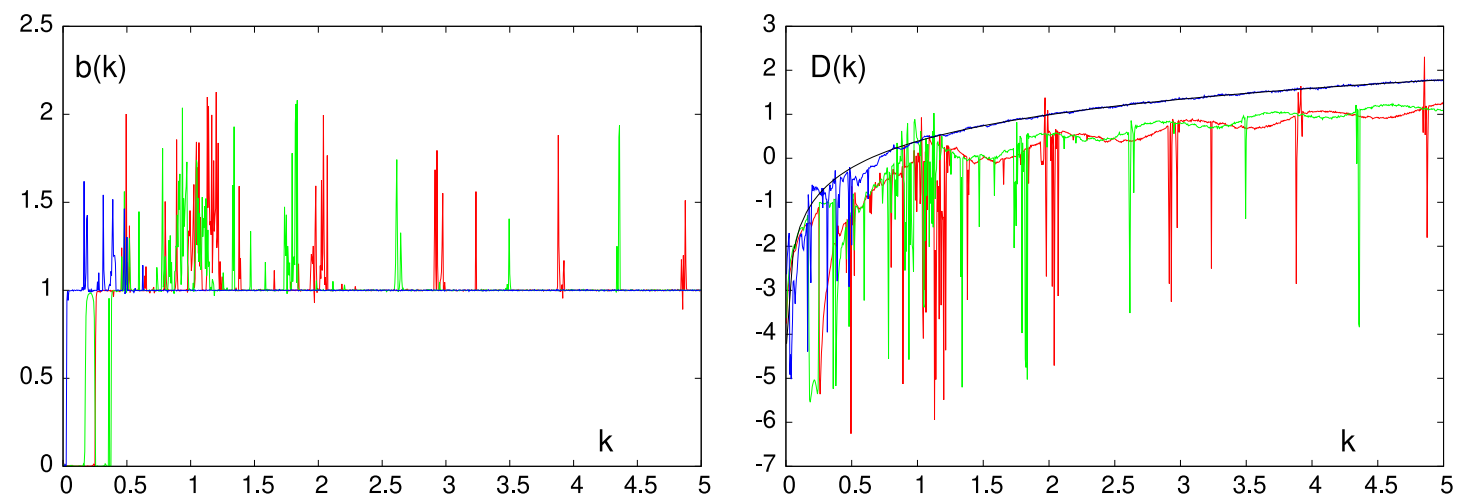

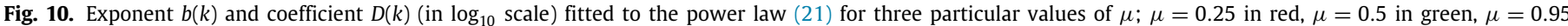

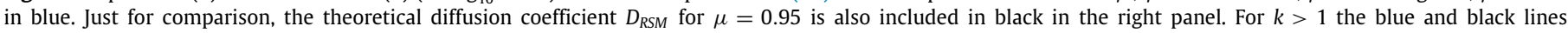
are almost identical. (For interpretation of the references to color in this figure legend, the reader is referred to the web version of this article.)

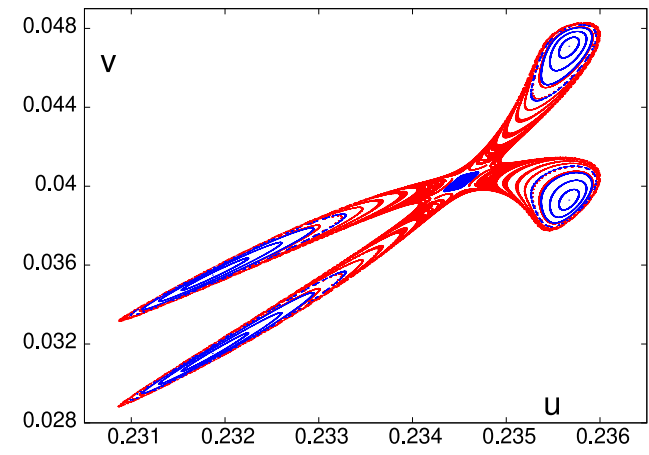

Fig. 11. Dynamics of the RSM for $k=1.835, \mu=0.5$ around a period 2 elliptic point that is created by bifurcation of the elliptic fixed point of the accelerator mode $|n|=2$. (For interpretation of the references to color in this figure legend, the reader is referred to the web version of this article.)

unstable at $k \approx 1.8219066$ leading to a 2-periodic elliptic orbit surrounded by invariant curves. For $k=1.835$ islands of period 4 exist around each one of two points of period 2 and the invariant curves that surrounded both points are destroyed. We take initial conditions near one of the points of period 2, say $P$, and iterate them. For moderate times the trajectories remain close to $P$ under the square of the map, but for large times they escape if we do not start very close to $P$. These orbits are depicted in Fig. 11 in red. On the other hand, if we take initial conditions close to $P$ and also close to the center of some of the islands of period 4 around it, the iterates remain bounded around the periodic points and they are drawn in blue. Of course, there is a similar pattern when we consider the image under the RSM, but we show just one of the parts. The point $P$ is located at the center of the very small set of blue curves near the center of the plot.

We see that although it would be expected a similar dynamics for all these nearby trajectories, those that appear in blue are bounded to a small neighborhood of the periodic points, while those in red, after a very large number of iterations, they escape.

\subsection{The Shannon entropy}

In order to show up all periodic solutions of the RSM for large $k$, following $[8,21]$ we take advantage of the Shannon entropy to measure phase correlations. Theoretical background on the Shannon entropy can be found, for instance, in [22,23]. However a different approach to the entropy in dynamical systems is presented for example in [24].
Let us summarize here the analytical formulation applied to the RSM defined in the torus $\mathbb{T}^{2}$, i.e., $x, y \in B=\mathbb{S}$. Let $\alpha$ be a partition of $B$,

$\alpha=\left\{a_{i} ; i=1, \ldots, q\right\}$,

a collection of $q$ intervals or one-dimensional cells that cover $B$. The elements $a_{i}$ are assumed to be measurable and disjoint.

For a given finite trajectory $\gamma=\left\{\left(y_{i}, x_{i}\right) \in B \times B, i=1, \ldots, N\right\}$ of the RSM, let $\gamma_{x}=\left\{x_{i} \in B, i=1, \ldots, N\right\} \subset \gamma$. If $n_{k}$ denotes the number of phase values in $a_{k}$, then its measure is $n_{k} / N$ and the entropy reads

$S\left(\gamma_{x}, \alpha\right)=-\sum_{k=1}^{q}\left(\frac{n_{k}}{N}\right) \ln \left(\frac{n_{k}}{N}\right)=\ln N-\frac{1}{N} \sum_{k=1}^{q} n_{k} \ln n_{k}$.

For the partition (23) and any $\gamma_{x}$ it is $0 \leq S\left(\gamma_{x}, \alpha\right) \leq \ln q$. The minimum occurs when all the phase values are restricted to a single element of $\alpha$ implying full phase correlation and thus $S=0$. On the other hand, the maximum entropy appears when all the elements have the same measure, $N / q$, therefore $S=\ln q$.

In $[8,21]$ it was shown that if $n_{k}$ follows a Poissonian distribution with mean $N / q \gg 1$, then the entropy (24) for uncorrelated motion, say $\gamma_{x}^{r}$, reduces to

$S\left(\gamma_{x}^{r}, \alpha\right) \approx \ln q-\frac{q}{2 N}$.

Since we are dealing with the entropy of the phase values of a given orbit of the RSM for large $k$, it is expected that all the elements of the partition have a positive measure. ${ }^{1}$

Notice that for random motion $|S-\ln q|=\mathcal{O}\left(N^{-1}\right)$ being $N \gg 1$ the total number of iterates of the map, so for any finite partition such that $q / N \ll 1$ the entropy reduces to

$S\left(\gamma_{x}^{r}, \alpha\right) \approx \ln q$.

Assuming that the approximation (25) partially holds for any chaotic trajectory $\gamma$ but $n_{k}$ following a different non-Poissonian distribution, the entropy of $\gamma_{x}$ can be written as

$S\left(\gamma_{x}, \alpha\right) \approx \ln q-\frac{q}{2 N} R, \quad R \geq 1$,

and if $\gamma_{x}$ presents weak phase correlations, $R \approx 1$.

Introducing the information as

$\mathcal{I}\left(\gamma_{x}, \alpha\right)=1-\frac{S\left(\gamma_{x}, \alpha\right)}{\ln q}$,

\footnotetext{
1 In case of a partition in phase space, $B \times B$, for any trajectory $\gamma$, except for random motion, only $q_{0}<q$ cells will have non-zero measure.
} 

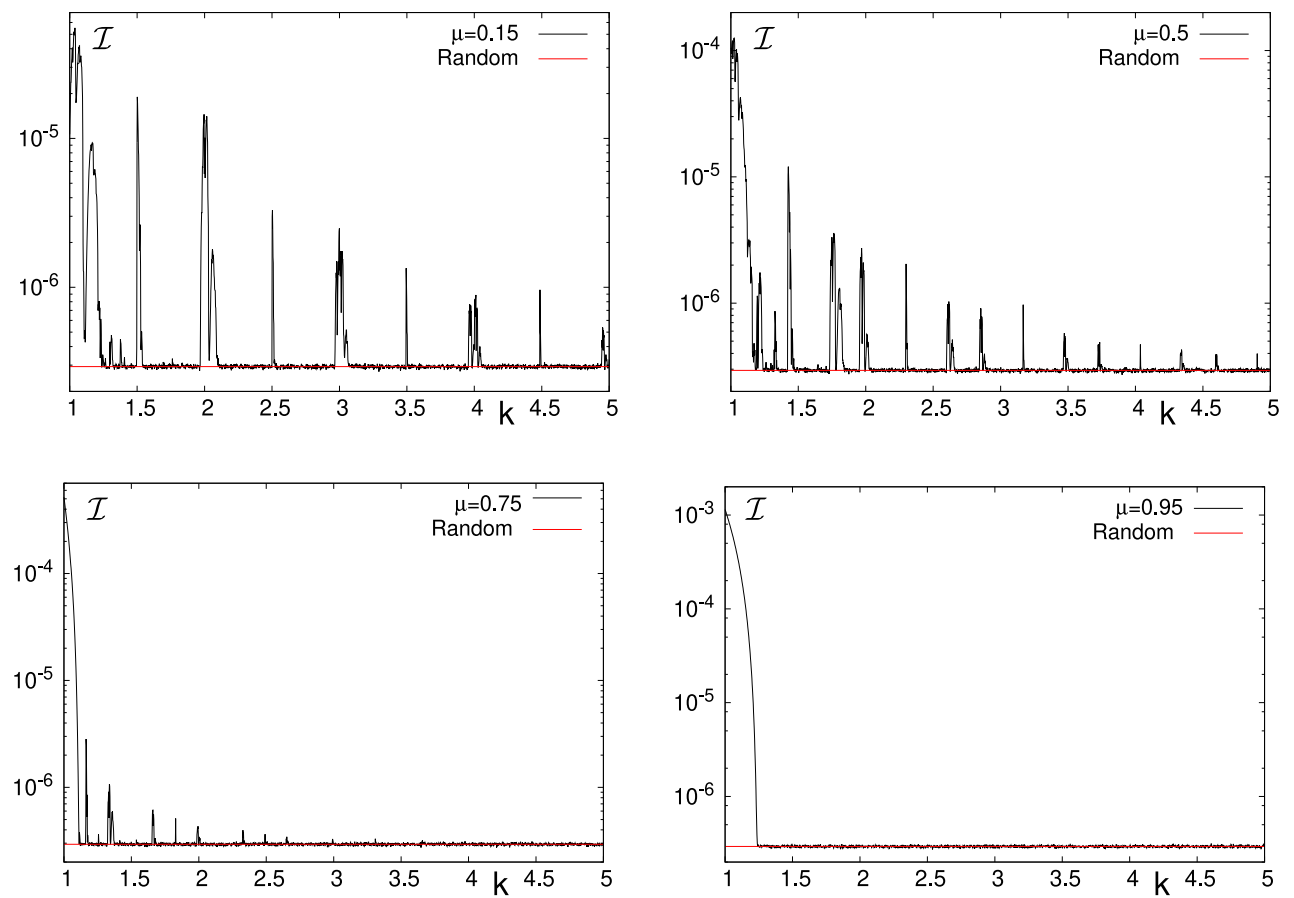

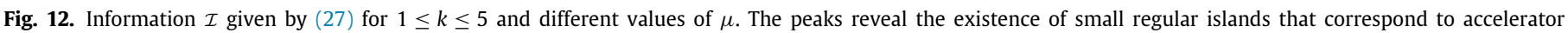
modes or high periodic solutions. The location of the peaks agrees with (10).

and using (25) and (26) the information becomes

$$
\begin{aligned}
& \mathcal{I}^{r} \equiv \mathcal{I}\left(\gamma_{x}^{r}, \alpha\right) \approx \frac{q}{2 N \ln q}>0, \\
& \mathcal{I}\left(\gamma_{x}, \alpha\right) \approx \frac{q R}{2 N \ln q}=R \mathcal{I}^{r},
\end{aligned}
$$

Therefore the information thus defined measures deviations of chaotic motion in the RSM with respect to a random system. This result is the key point in revealing periodic solutions and/or accelerator modes in the RSM.

In [8] the same approach was introduced to investigate periodic solutions in the SM for large $k$, computing the entropy not only for the phases but for the full trajectory on phase space and in any case the analytical and numerical results were quite similar.

We compute then the Shannon entropy/information (27) of the phase values of the RSM for an ensemble of $n_{p}=1000$ random initial conditions centered at $(u, v)=\left(10^{-4}, 10^{-4}\right)$ of size $10^{-4}$ for $N=10^{6}$ iterates. The parameters are such that $k \in[1,5]$ with $\Delta k=0.0005$ and four different $\mu$ values. Since in the estimates given in (28) $N$ denotes the total number of iterates, in the present experiments $N \rightarrow n_{p} N$. We adopt a partition of $q=5000$ cells of the unit interval, so the condition $n_{p} N / q \gg 1$ clearly holds.

The results are shown in Fig. 12 where we include the expected value for random motion where the information is given by $\mathcal{I}^{r}$ defined in (28) and corresponds to a lower bound for the information that, for the adopted values of the parameters, is $\mathcal{I}^{r} \approx 3 \times 10^{-7}$. For comparatively small values of $\mu$ the peaks arising at $k(1+\mu) \approx|n|,(2|n|+1) / 2$ could be identified, as in the $\mathrm{SM}$, with accelerator modes of period one and four respectively. Some other peaks would correspond to trajectories of period two.

As discussed above, accelerator modes of period one appear for $(k, \mu)$ in the interval $n^{2}<k^{2}\left(1+\mu^{2}\right)<n^{2}+4 / \pi^{2}$, valid for small $\mu$ (Fig. 2 provides an accurate stability interval for all $\mu$ ). Therefore for $k=|n|$ we can estimate a bound of $\mu$ for the existence of these periodic solutions, $\mu<\mu_{a}=2 /(|n| \pi) \approx 0.637 /|n|$, this value of $\mu_{a}$ is close to the actual threshold for $\mu$ that can be derived from Fig. 2 for instance for $k=1$. However very thin stability intervals arise at large values of $\mu, \mu \gtrsim 0.9$ that accumulate towards $\mu=1, k=0$.

For the $\mu$ values considered at the top panel of the figure, $\mu=0.15,0.5$, this inequality clearly holds in case of $\mu=0.15$ whenever $n \lesssim 4$. For $\mu=0.5$, the actual bounds are given in Fig. 2.

On the other hand, the center of the integer resonances are stable provided that $k \leq 4 / \pi$ and such that $\mu>\mu_{c}=\pi k / 2-1$. Thus for $k=|n|$ both conditions are fulfilled provided that $k=$ $|n| \approx 1$ and thus $\mu_{c}=\pi / 2-1 \approx 0.571$, larger than the $\mu$ values adopted in the two figures at the top panel.

The opposite situation occurs for the figures at the bottom panel where $\mu=0.75,0.95$, the center of the integer resonances become again stable but only for $k=1$ and $\mu_{c}<\mu<1$. Accelerator modes of period one only exist for $k<1$ and $\mu$ large enough.

In the figure corresponding to $\mu=0.75$, the large smooth maximum at $k=1$ is due to the stability domain of the integer resonance and the small peaks observed for $k>1$ are certainly due to high periodic solutions and/or accelerator modes as Fig. 3 reveals. Remarkably is the result for $\mu=0.95$, just a wide stable region arising at $k=1$ due to the integer resonance is the dominant structure, no other relevant stable periodic solutions seem to exist for $k>1$ as predicted.

Now let us compute the entropy/information fixing $k=1,2$ and $0 \leq \mu<1$, for $2000 \mu$ values and considering the same ensemble and parameters than in the previous experiments. The results are shown in Fig. 13 where we observe that for $k=1$ and $\mu<0.637$ period one accelerator modes should exist (as well as other high period solutions) and they would be responsible of the particular structure observed in $\mathcal{I}$ for $\mu<0$.6. For larger values, $\mu>0.68$ the information increases in a smooth way with $\mu$ as long as the integer resonance becomes wider. In the interval $0.571<\mu<0.637$ both accelerator modes for $k=1$ and the integer resonances coexist as stable domains and within a similar interval $0.5<\mu<0.68$, it is observed a transition between both regimes in the information. For $k=2$ one period accelerator 


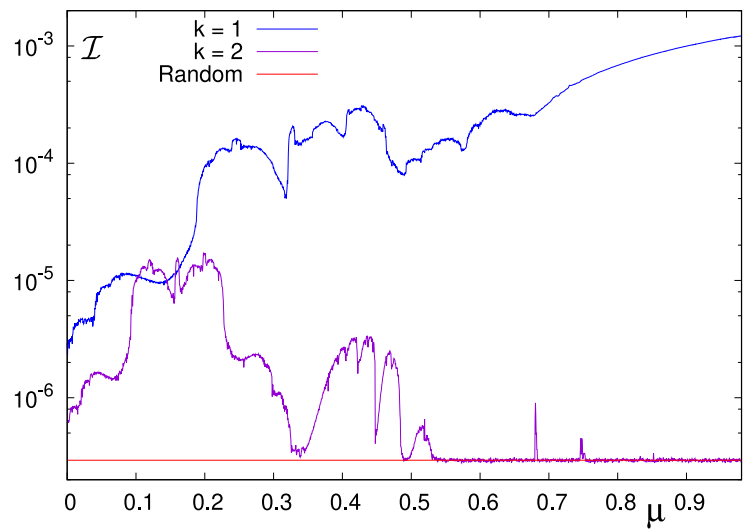

Fig. 13. $\mathcal{I}$ as a function of $\mu$ for two particular values of $k$. The red line corresponds to the random reference level given by $\mathcal{I}^{r}$. (For interpretation of the references to color in this figure legend, the reader is referred to the web version of this article.)

modes only exist for $\mu<\pi^{-1} \approx 0.32$ and this threshold value is observed in the figure. The remainder structures present in $\mathcal{I}$ should be attributed to high-periodic solutions of the RSM, since the integer resonances are destroyed for any $\mu$ at this value of $k$. Indeed, from Fig. 3 we see that period 2 solutions are stable for $k=2$ and $0.6 \lesssim \mu \lesssim 0.9$.

In order to visualize the phase space structure of the RMS for $k=1$ and $\mu=0.15,0.75$ revealed by $\mathcal{I}$, instead of plotting the iterates of some initial conditions as in Fig. 5, we proceed as follows. We consider $2000 \times 2000$ initial values of $0 \leq u, v<1$. Each initial condition is iterated under the RSM together with the differential map up to $N=100$ and the value of a fast dynamical indicator at $N=100$ is computed, in this case the conditional entropy (see [25] for details). The (symmetric) conditional entropy of nearby orbits, is such that it goes as $\delta_{0}^{2} \lambda^{2} N^{2} \ll 1$ for regular, stable motion, where $\lambda$ is the linear rate of divergence of nearby quasi-periodic orbits and $\delta_{0} \ll 1$, is the initial of the norm of the deviation vector. On the other hand, it behaves as $\delta_{0}^{2} \exp (2 \sigma N)$ for chaotic motion, where $\sigma$ is the maximum Lyapunov exponent of a given trajectory. This fast dynamical indicator turns out to be very useful when considering small number of iterates since it is quadratic in the solution of the variational equations.

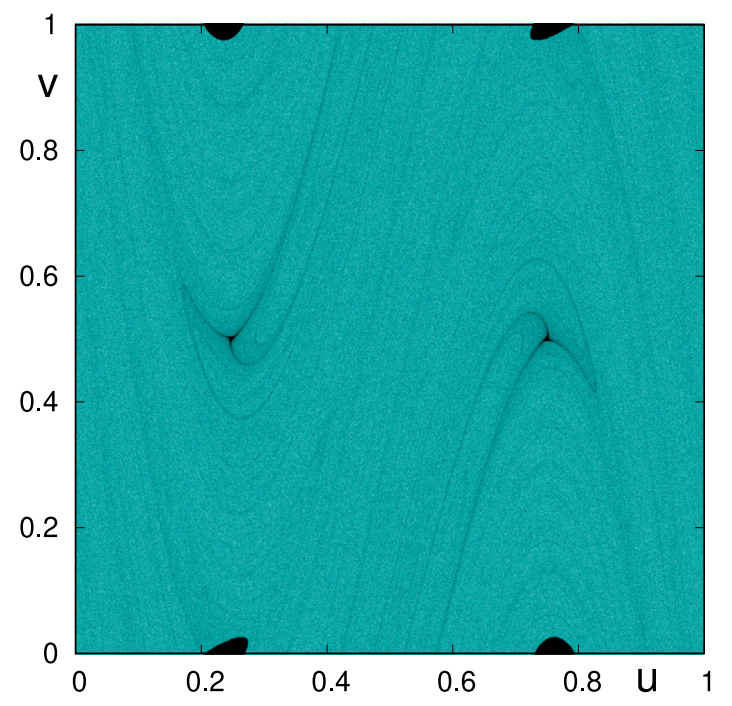

Fig. 14 presents contour plots of the conditional entropy where light blue colors denote highly chaotic, almost random motion while dark blue represents stability. In the figure at the left, where $\mu=0.15<\mu_{c}$, the most relevant stable domains are the one-period accelerator modes around $v=0,1$ and the period two trajectory centered at $v_{0}=0.5, u_{0}=0.25,0.75$, the latter values, after the scaling $u \rightarrow x$, are the solutions of (15) for $k=1, \mu=0.15$. For $x_{0}=\pi / 2,3 \pi / 2\left(u_{0}=0.25,0.75\right)$ the stability interval (16) reduces to $k \mu<2 / \pi$ and thus the centers are stable for $k=1$ and $\mu<0.637$. On the other hand, for the period two orbit at $v_{0}=0.5, u_{0}=0,0.5$ one has instability as follows from (16).

As mentioned, the period 2 orbit which appears at $(0.25,0.5)$ and $(0.75,0.5)$ is stable. For concreteness consider the point $P=$ $(0.25,0.5)$. Around $P$ one finds invariant curves such that moving away from $P$ they approach a last curve which is close to a rightangled triangle. Close to what could be seen, in some sense, at the vertices of this triangle there are three points in a period-3 unstable orbit around $P$ (under the square of the map, of course). Globally one has a period-6 unstable orbit. One of the points on it is $\approx(0.25586307,0.50308448)$. This orbit is hyperbolic and the filaments that one can guess in the plot, tending to $(0.25,0.5)$ ( 3 of them) and to $(0.75,0.5)$ (the other 3$)$, are nothing else that points rather close to the branches of the stable manifold of the period- 6 orbit. They appear in dark colors because most of the iterates remain close to the period- 6 orbit.

In the figure at the right, that corresponds to $\mu=0.75>\mu_{c}$, we observe a large domain of stable motion that corresponds to the integer resonances, since $k<2(1+\mu) / \pi$. On the other hand period one accelerator modes do not exist because $\mu>\mu_{a}$. The observed small islets of stability are due to high periodic solutions of the map as revealed by Fig. 3 .

Thus all these "ordered" dynamical structures that appear in the phase space of the RSM for these particular values of the parameters induce strong correlations that was measured by the entropy.

As an additional information, we mention that in case of $k=$ $1, \mu=0.5$, starting from $P=(0.222,0.5)$ after $10^{9}$ iterations of the map, the iterates cover completely the chaotic component while if instead we follow the iterates from $Q=(0.223,0.5)$, invariant curves appear around the 2-periodic trajectory. For any starting point lying outside the stability zones, the corresponding iterates also fill up almost uniformly the full chaotic region. For a

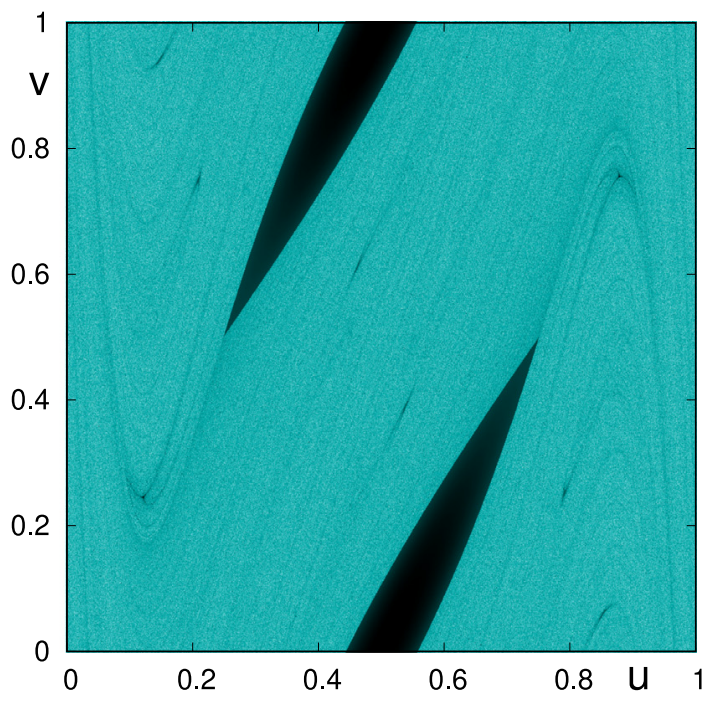

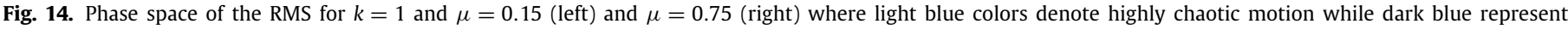
stable motion. (For interpretation of the references to color in this figure legend, the reader is referred to the web version of this article.) 
grid of $200 \times 200$ pixels, 680 of them appear empty (stable zones), 546 present only a few iterates (around the stable zones) while in the remainder 38774 pixels, the average number of iterates is 25594.3 with a standard deviation of 161.3. In case of $\mu=0.6$ a similar behavior is observed but for $P=(0.225,05)$ and $Q=$ $(0.227,0.5)$.

Therefore we observe that the phase space of the RSM for large values of $k$ is nearly ergodic whenever the latter takes values outside of any stability interval. On the other hand, if $k$ adopts values within a stability interval the phase space presents two components, a small one corresponding to islets of stability and a large chaotic component with a nearly uniform distribution.

\section{Conclusions}

Along this paper we investigate the dynamics of the RSM. We show that this system has many differences with respect to the SM. Indeed, its associated Hamiltonian possesses all the Fourier spectrum at order $K$ in both $x$ and $t$, with bounded amplitudes that depend on $\mu$. This fact leads to several unexpected consequences. For instance, most of the resonances are stable for comparatively large values of $K$ and therefore the system could be well approximated by an integrable one for suitable values of $\mu$. We also have, among many other peculiarities, that for moderate values of $\mu$ RICs exist for values of $K$ much larger than for the SM and even for the SM with two harmonics.

On the other hand for large values of $K$, even if $\mu$ has a moderate value, and for $\mu$ close to 1 , even if $K$ is moderate, the RSM approaches an ergodic system.

All results are supported by analytical estimates as well as many numerical experiments, some of them required quadruple precision. The implementation of the Shannon entropy to measure phase correlations allow us to show the location of all periodic solutions and therefore to get a clear picture of the dynamics of the RSM in the chaotic regime.

\section{CRediT authorship contribution statement}

Pablo M. Cincotta: Conceptualization, Methodology, Software, Formal analysis, Writing - original draft, Writing - review \& editing.

\section{Declaration of competing interest}

The authors declare that they have no known competing financial interests or personal relationships that could have appeared to influence the work reported in this paper.

\section{Acknowledgments}

P.M.C. was supported by grants from Consejo Nacional de Investigaciones Científicas y Técnicas de la República Argentina (CONICET), the Universidad Nacional de La Plata, Argentina and Instituto de Astrofísica de La Plata, Argentina. C.S. was supported by grants 2017-SGR-1374 (Catalonia) and MTM2016-80117-P (Spain). He also thanks J. Timoneda for maintaining the computing facilities of the Group of Dynamical Systems of the Universitat de Barcelona, that have been largely used in this work. The two anonymous reviewers are also acknowledged for their criticism that helped us to improve this manuscript.

\section{Appendix. The $\delta$ distribution and related topics}

Assume we have a simple map of the form

$\bar{y}=y+f(x), \quad \bar{x}=x+\bar{y}$,

being $f$ a $2 \pi$-periodic function. It can be seen as one of the possible discretizations of the flow $\dot{x}=y, \dot{y}=f(x)$, Hamiltonian in the present case, with Hamiltonian $H(x, y)=y^{2} / 2-h(x)$, being $h$ a primitive of $f$. To try to represent the map as the time- $2 \pi$ flow of a Hamiltonian we can use a periodic $\delta$ distribution. We denote as $\delta_{2 \pi}(t)$ the distribution which consists on the sum of $\delta$ distributions at all the multiples of $2 \pi$. Then we can rewrite the equations as $\dot{x}=y, \dot{y}=f(x) \delta_{2 \pi}(t)$. Note that if at $t=0$ the values of $(x, y)$ are $\left(x_{0}, y_{0}\right)$, we want to have the image $\left(x_{1}, y_{1}\right)$ under the map as $y_{1}=y_{0}+f\left(x_{0}\right), x_{1}=x_{0}+y_{1}$. Using $\dot{y}=f(x) \delta_{2 \pi}(t)$ produces the desired jump in $y$, but then $x$ is increasing with velocity $y_{1}$ during time $2 \pi$ instead of time 1 . We can modify the equations and the Hamiltonian as

$\dot{x}=\frac{y}{2 \pi}, \quad \dot{y}=f(x) \delta_{2 \pi}(t), \quad H(x, y, t)=\frac{y^{2}}{4 \pi}-h(x) \delta_{2 \pi}(t)$.

Why are we interested in introducing $\delta_{2 \pi}(t)$ ?

The idea is that we can use the Fourier expansions of $h(x)$ (or of $f(x)$ ) and of $\delta_{2 \pi}(t)$ to try to identify resonances. This is used in Section 3 to compare the amplitudes of the resonances in some numerical examples. To this end we recall the expansion

$\delta_{2 \pi}(t)=\frac{1}{2 \pi}+\frac{1}{\pi} \sum_{m=1}^{\infty} \cos (m t)=\frac{1}{2 \pi} \sum_{m=-\infty}^{\infty} \cos (m t)$.

We recall that for the RSM, the functions $f$ and $h$, that were denoted as $f(x ; \mu)$ and $K V_{0}(x ; \mu)$, are

$f(x ; \mu)=K \frac{\sin x}{1-\mu \cos x}, \quad V_{0}(x ; \mu)=-\frac{1}{\mu} \ln (1-\mu \cos x)$,

last one for $\mu \neq 0$. As $V_{0}(x ; \mu)$ is an even function, the Fourier expansion only contains cosines functions and a constant term, which is irrelevant. Concretely we can write

$V_{0}(x ; \mu)=\sum_{l=1}^{\infty} C_{l}(\mu) \cos (l x)$.

Proposition. For $l \geq 1$ one has

$C_{l}(\mu)=\frac{2 \mu^{l-1}}{l\left(1+\sqrt{1-\mu^{2}}\right)^{l}}$

for the coefficients in (30).

Proof. According to [20] p. 391, for $a^{2}<1, n \geq 1$ one has

$\int_{0}^{\pi} \frac{\sin n x \sin x}{1-2 a \cos x+a^{2}} d x=\frac{\pi}{2} a^{n-1}$

Using $a=\mu /\left(1+\sqrt{1-\mu^{2}}\right)$ one obtains the coefficients of the expansion for $f(x ; \mu) / K$ and then, by integration, the values in (31) follow.

Multiplying $V_{0}(x ; \mu)$ by $\delta_{2 \pi}(t)$ one obtains the potential $V(x, t$; $\mu$ ) given in (5).

\section{Remarks.}

- We note that the first coefficient of $\sin x /(1-\mu \cos x)$ is $C_{1}(\mu)=2 /\left(1+\sqrt{1-\mu^{2}}\right)$ while the lth coefficient is $C_{1}(\mu)\left(\mu /\left(1+\sqrt{1-\mu^{2}}\right)\right)^{l-1}$. That is, they decrease geometrically. As an example, the maximum of $\sin x /(1-\mu \cos x)$ is equal to $1 / \sqrt{1-\mu^{2}}$ and is attained when $x=x^{*}=$ 
$\cos ^{-1}(\mu), x^{*} \in(0, \pi / 2)$. If we want to see how many terms are needed to have in $x^{*}$ a value larger than 0.99 of the maximum, one needs 5,16 and 51 terms for $\mu=0.9,0.99$ and 0.999 , respectively. This is in contrast with the SM with two harmonics, where depending of one parameter the ratio is arbitrary. See, e.g., [17] Section 6.

- It is worth to comment on what happens if we use a truncation of the Fourier series of $\delta_{2 \pi}(t)$ and integrate the corresponding ODE. This allows to use standard integration routines with step-size control. Let us denote as $\delta_{2 \pi}^{M}(t)$ the truncation of the series using $|m| \leq M$. It is easy to obtain the identity

$\delta_{2 \pi}^{M}(t)=\frac{1}{2 \pi} \sum_{m=-M}^{M} \cos (m t)=\frac{1}{2 \pi} \frac{\sin ((2 M+1) t / 2)}{\sin (t / 2)}$

if $t \neq 0$ and which tends to the maximum $(2 M+1) /(2 \pi)$ when $t \rightarrow 0(\bmod 2 \pi)$. In $[0,2 \pi]$ it decreases from the maximum to zero in $[0,2 \pi /(2 M+1)]$ and increases from 0 to the maximum in $[2 \pi 2 M /(2 M+1), 2 \pi]$. The function is bounded by the curves $1 /(2 \pi \sin (t / 2))$ and $-1 /(2 \pi \sin (t / 2))$, being tangent to them at the points of the form $(2 k+1) \pi /(2 M+$ $1), k=0, \ldots, 2 M$ for $k$ even and odd, respectively. For $M$ large the function has fast oscillations between the two zeros mentioned above.

But looking at the time- $2 \pi$ map, when $t$ goes from 0 to $2 \pi$, in the ODE $\dot{x}=y, \dot{y}=f(x) \delta_{2 \pi}^{M}(t)$ half of the change in $y$ comes around $t=0$, essentially nothing until the vicinity of the last zero before $2 \pi$, and the other half of the change comes around $t=2 \pi$. Hence the time- $2 \pi$ map in that case can be written as $(x, y) \mapsto(\bar{x}, \bar{y})$ where, approximately,

$y_{\text {aux }}=y+f(x) / 2, \quad \bar{x}=x+y_{a u x}=x+y+f(x) / 2$,

$\bar{y}=y+f(x) / 2+f(x+y+f(x) / 2) / 2$,

which can be far from what we want.

A possible solution consists in having the total jump of $y$ at the beginning. For this we can apply a shift to $t$ in $\delta_{2 \pi}^{M}(t)$. We can look for a value of $s>0$, close to zero, such that $\int_{-s}^{s} \delta_{2 \pi}^{M}(t) d t=1$. Then the jump in $y$ near $t=0$ is close to $f(x)$ getting $\bar{y}$ and, as $\int_{s}^{2 \pi-s} \delta_{2 \pi}^{M}(t) d t=0$ no more changes are produced until $t=2 \pi-s$. In a similar way the speed of change of $x$ is, approximately, $\bar{y} /(2 \pi)$ in the range $[-s, 2 \pi-s]$ and we get $\bar{x}=x+\bar{y}$.

Hence, the equation for $\dot{y}$ can be changed to $\dot{y}=f(x) \delta_{2 \pi}^{M}(t-$ $s)$. It is clear that $s$ depends on $M$ but one can check that the product $s(2 M+1)$ tends to $\approx 3.8529$ when $M \rightarrow \infty$, with errors $\mathcal{O}\left(M^{-2}\right)$. As an example, we use $(\mu, K)=(0.978,0.042)$ like in Fig. 4 right, and start at $(x, y)=(0,0.07 \times 2 \pi)$. After $10^{8}$ iterates and keeping data after every 1000 iterates, the plots using iterations of the map and of the time- $2 \pi$ flow, with $M=50$, look almost identical. The only differences are small variations in the density of points.

As an additional example we used $K=1, \mu=0.1,0.2, \ldots$, 0.9 and a $100 \times 100$ lattice for $(x, y) \in[0,2 \pi)^{2}$. Comparing iterations of the map and the time- $2 \pi$ flow one has maxi- mal differences of the order of $10^{-3}$. It has been checked for values of $M$ equal to several hundreds.

\section{References}

[1] B.V. Chirikov, Institute of Nuclear Physics, Novosibirsk (in Russian), Preprint 267 (1969), Engl. Transl. CERN Trans. 71-40, Geneva, (1971). Available at http://www.quantware.ups-tlse.fr/chirikov/publclass.html.

[2] B.V. Chirikov, A universal instability of many-dimensional oscillator systems, Phys. Rep. 52 (1979) 263.

[3] J.D. Meiss, Symplectic maps, variational principles and transport, Rev. Modern Phys. 64 (1992) 795.

[4] I.I. Shevchenko, The dynamical temperature and the standard map, Physica A 386 (2007) 85

[5] N. Miguel, C. Simó, A. Vieiro, On the effect of islands in the diffusive properties of the standard map, for large parameter values, Found. Comput. Math. 15 (2014) 89.

[6] T. Manos, M. Robnik, Survey on the role of accelerator modes for anomalous diffusion: The case of the standard map, Phys. Rev. E 89 (2014) 022905.

[7] M. Harsoula, G. Contopoulos, Global and local diffusion in the standard map, Phys. Rev. E 97 (2018) 022215.

[8] P.M. Cincotta, I.I. Shevchenko, Correlations in area preserving maps: a Shannon entropy approach, Physica D (2020) 132235

[9] G. Gómez, J.M. Mondelo, C. Simó, A collocation method for the numerical fourier analysis of quasi-periodic functions, I: Numerical test and examples, Discrete Contin. Dyn. Syst. B 14 (2010) 41.

[10] P.M. Cincotta, C.M. Giordano, C. Simó, Phase space structure of multidimensional systems by means of the mean exponential growth factor of nearby orbits, Physica D 182 (2003) 151-178.

[11] P.M. Cincotta, C.M. Giordano, Theory and applications of the mean exponential growth factor of nearby orbits (MEGNO) method, Lect. Notes Phys. 915 (2016) 93.

[12] M. Guzzo, E. Lega, C. Froeschlé, A numerical study of arnold diffusion in a priori unstable systems, Comm. Math. Phys. 290 (2009) 557.

[13] V.I. Arnold, On the nonstability of dynamical systems with many degrees of freedom, in: Soviet Mathematical Doklady 5, 1964, pp. 581-585.

[14] J.A. Ketoja, R.S. MacKay, Fractal boundary for the existence of invariant circles for area-preserving maps: observations and renormalisation explanation, Physica D 35 (1989) 318.

[15] J.A. Ketoja, R.S. MacKay, Rotationally-ordered periodic orbits for multiharmonic area-preserving twist maps, Physica D 73 (1994) 388.

[16] A. Fox, J.D. Meiss, Critical invariant circles in asymmetric and multiharmonic generalized standard maps, Commun. Non-linear Sci. Numer. Simul. 19 (2014) 1004.

[17] R. Martínez, C. Simó, Return maps dynamical consequences and applications, Qual. Theory Dyn. Syst. 14 (2015) 353.

[18] A. Giorgilli, V.F. Lazutkin, Some remarks on the problem of ergodicity of the standard map, Phys. Lett. A 272 (2000) 359.

[19] I.I. Shevchenko, Isentropic perturbations of a chaotic domain, Phys. Lett. A 333 (2004) 408.

[20] I.S. Gradshteyn, I.M. Ryzhik, Table of Integrals, Series, and Products, seventh ed., Elsevier/Academic Press, Amsterdam, 2007.

[21] P.M. Cincotta, C.M. Giordano, Phase correlations in chaotic dynamics: a Shannon entropy measure, Celestial Mech. Dynam. Astronom. 130 (2018) 74.

[22] C. Shannon, W. Weaver, The Mathematical Theory of Communication, Illinois U.P, Urbana, 1949.

[23] A. Lesne, Shannon entropy: a rigorous notion at the crossroads between probability, information theory, dynamical systems and statistical physics, Math. Struct. Comput. Sci. 24 (2014) e240311.

[24] V. Arnol'd, A. Avez, Ergodic Problems of Classical Mechanics, second ed. Addison-Wesley, New York, 1989.

[25] P.M. Cincotta, C. Simó, Conditional entropy, Celestial Mech. Dynam. Astronom. 73 (1999) 195, http://dx.doi.org/10.1016/j.physd.2019.132235. 